\title{
Anomalous chiral edge states in spin-1 Dirac quantum dots
}

\author{
Hong-Ya $\mathrm{Xu}^{1}$ and Ying-Cheng Lai ${ }^{1,2, *}$ \\ ${ }^{1}$ School of Electrical, Computer, and Energy Engineering, Arizona State University, Tempe, Arizona 85287-5706, USA \\ ${ }^{2}$ Department of Physics, Arizona State University, Tempe, Arizona 85287, USA
}

(Received 28 June 2019; published 17 January 2020)

\begin{abstract}
We uncover an unexpected family of in-gap chiral edge states in noninverted spin-1 Dirac quantum dots. The system represents a topologically trivial confinement configuration where such edge states are not expected according to the conventional wisdom. In particular, for a massive type of confining potential, two distinct situations can arise: with or without mass sign change, corresponding to a quantum dot with or without band inversion, respectively. The former case is conventional, where topologically protected chiral edge modes can arise in the gap. For the latter, contrary to the belief that there should be no one-way current-carrying edge channels, we find the surprising emergence of such edge states and the spin- 1 analog of Majorana modes. These states are strikingly robust and immune to backscattering. In the presence of a magnetic field, the edge states result in peculiar Fock-Darwin states originated from Landau-level confinement. The unexpected phenomenon is also validated in systems with bulk-topology regularization through (1) a properly regularized continuum model and (2) an experimentally accessible tight-binding dice lattice system.
\end{abstract}

DOI: 10.1103/PhysRevResearch.2.013062

\section{INTRODUCTION}

A fundamental principle in the study of topological phases of matters is the bulk-edge correspondence [1,2], which states that robust interfacial modes immune to impurities or geometric perturbations can arise on the boundary between domains with distinct bulk topological invariants. The emergence of the interfacial states thus represents a topological confinement mechanism with applications in controlling a variety of wave phenomena. The principle was originally discovered in electronic systems, but in recent years topological states and the bulk-edge correspondence have been extended to a broad range of fields in physics, from cold atoms [3], optics and photonics [4], to classical fluid and solid mechanics [5]. Generally, the Dirac theory of electrons provides a theoretical framework to analyze and understand the topological interfacial states. For example, in one spatial dimension, a sign-changing kink profile of the mass term can lead to topological confinement of zero-energy bound states with the peculiar property of fractional charge [6]. In higher dimensions, an extension of the kink-shaped mass profile can give rise to distinct topological excitations such as vortices and magnetic monopoles [7]. In gapped Dirac materials [8-10] such as magnetic topological insulators, a mass term in the Dirac equation corresponds to a bulk band gap while its sign determines the band arrangement order and the resulting bulk topological invariant. Experimentally, the sign-changing Dirac mass scenario can be implemented using domain-wall junctions with band inversion in Dirac materials.

\footnotetext{
*Ying-Cheng.Lai@asu.edu

Published by the American Physical Society under the terms of the Creative Commons Attribution 4.0 International license. Further distribution of this work must maintain attribution to the author(s) and the published article's title, journal citation, and DOI.
}

The focus of our work is on two-dimensional Dirac materials hosting the pseudospin-1 generalization of Dirac fermions (hereinafter referred to as spin-1 Dirac particles). Massless spin-1 Dirac particles arise in systems whose energy band structure constitutes a pair of Dirac cones and a flat band, with a conical intersection of triple degeneracy [11-38]. Pseudospin-1 Dirac materials have been realized or simulated in a variety of physical systems ranging from photonic crystals [17] and optical lattices [27] to two-dimensional electronic lattices [36,37] and superconducting qutrit [38] as well as crystalline solids [31]. Quite recently, quantum materials hosting a flat band, such as the magic-angle twisted bilayer graphene, have become a forefront area of research. These materials can generate remarkable physical phenomena such as unconventional superconductivity [39,40], orbital ferromagnetism [41,42], and Chern insulating behavior with topological edge states.

In this paper, we present our discovery of the emergence of a class of anomalous in-gap chiral edge states without requiring the topological restriction dictated by conventional rules of either the index theorem or the bulk-edge correspondence. Our setting is the generic mesoscopic structure of quantum dot for massive spin-1 Dirac particles arising in materials with an energy gap. In particular, we consider confining potentials of the massive type with closed boundaries and ask the question of what type of topological quantum states may arise in such a confinement. As for spin- $\frac{1}{2}$ fermions $[43,44]$, the sign of the generalized spin-1 Dirac mass effectively defines the associated bulk topological invariant. There are two distinct situations of confinement: with or without a sign-changing mass. The corresponding topological invariant thus describes a quantum dot system with or without an inverted band alignment. The former case is conventional and has been known to host topologically protected chiral edge modes in the gap $[45,46]$. For the latter case where there is no band 


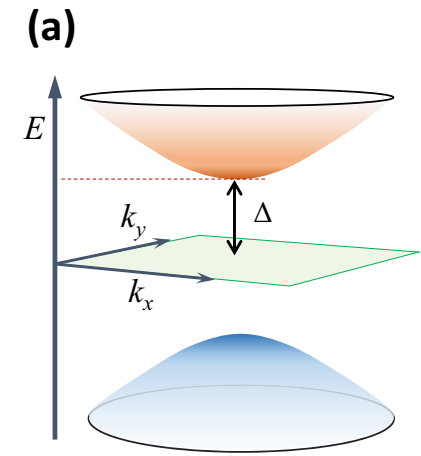

(b)

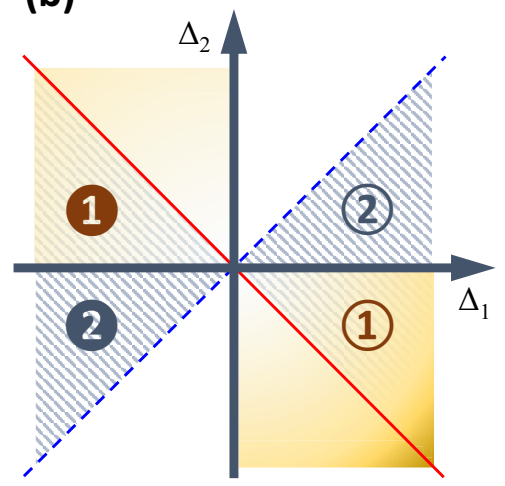

(c)
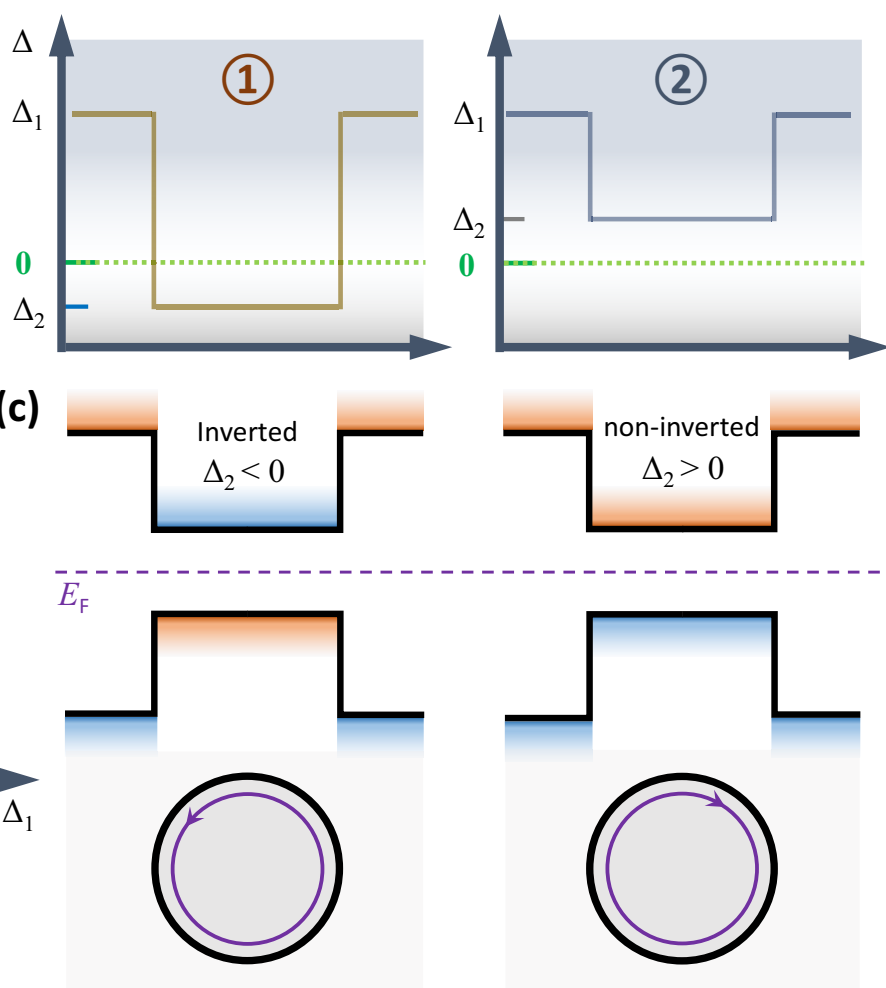

FIG. 1. Schematic illustration of confinement configuration and unconventional chiral edge states. The anomalous topological states are harbored at the closed interface delineating two insulating regions without band inversion. (a) Energy-momentum dispersion relation of a bulk massive spin-1 excitation, (b) phase diagram in the $\left(\Delta_{1}, \Delta_{2}\right)$ plane, and (c) the two distinct cases of confining configurations indicated in (b): with or without band inversion.

inversion and hence no change in the associated bulk topology, the expectation was that no current-carrying topological edge channels would arise. However, in sharp contrast to the conventional wisdom, we find that such states can arise for spin-1 Dirac particles and are remarkably robust against geometric perturbations.

\section{RESULTS FROM SYSTEMS DESCRIBED BY CONTINUUM DIRAC HAMILTONIAN}

The Hamiltonian for a two-dimensional (2D) spin-1 Dirac particle in the position representation is $\hat{H}=v_{F} \hat{\boldsymbol{S}} \cdot \hat{\boldsymbol{D}}+$ $\hat{S}_{z} \Delta(\boldsymbol{r})$, where $v_{F}$ is the Fermi velocity, $\hat{\boldsymbol{S}}=\left(\hat{S}_{x}, \hat{S}_{y}\right)$ and $\hat{S}_{z}$

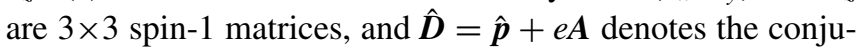
gate momentum with vector potential $\boldsymbol{A}$ defining an applied magnetic field $\boldsymbol{B}$. The second term in the Hamiltonian, where $\Delta(\boldsymbol{r})$ is the mass profile, is responsible for the confinement, which takes into account Klein tunneling. The Hamiltonian $\hat{H}$ acts on the three-component spinor wave function $\Psi(\boldsymbol{r})=$ $\left[\psi_{1}, \psi_{2}, \psi_{3}\right]^{T}$ with eigenenergy $E$. The associated homogeneous bulk spectra of $\hat{H}$ (with $\boldsymbol{A}=0$ and a given positive constant mass $\Delta$ ), as shown in Fig. 1(a), are $E_{0}=0$ and $E_{ \pm}= \pm \sqrt{v_{F}^{2} \hbar^{2} k^{2}+\Delta^{2}}$, with the corresponding (normalized) eigenstates given by

$$
\Psi_{k, 0}(\boldsymbol{r})=\frac{1}{|\epsilon|}\left(\begin{array}{c}
k_{-} / \sqrt{2} \\
-\delta \\
-k_{+} / \sqrt{2}
\end{array}\right) e^{i \boldsymbol{k} \cdot \boldsymbol{r}}
$$

and

$$
\Psi_{k, s}(\boldsymbol{r})=\frac{1}{2} \frac{k}{|\epsilon|}\left(\begin{array}{c}
k_{-} /(\epsilon-\delta) \\
\sqrt{2} \\
k_{+} /(\epsilon+\delta)
\end{array}\right) e^{i k \cdot \boldsymbol{r}},
$$

where the band index is $s=\operatorname{sign}\left(E_{ \pm}\right), k_{ \pm}=k_{x} \pm i k_{y}$, and the wave vector $\boldsymbol{k}=\left(k_{x}, k_{y}\right)$ has length $k=\sqrt{\epsilon^{2}-\delta^{2}}$ with $\epsilon=E / \hbar v_{F}$ and $\delta=\Delta / \hbar v_{F}$, which makes an angle $\theta=$ $\arctan \left(k_{y} / k_{x}\right)$ with the $x$ axis. The finite mass $\Delta$ accounts for the gap opening between the positive and negative energy bands, breaks the time-reversal symmetry $\mathcal{T}$, but preserves the antiunitary particle-hole symmetry $\mathcal{P}$. As such, the system falls into the same symmetry category as that of the Bogoliubov-de Gennes Hamiltonian for $p+i p$ superconductors hosting chiral/unpaired Majorana modes [47]. With the definition of the helicity operator $\hat{h}=\hat{\boldsymbol{S}} \cdot \boldsymbol{p} /|\boldsymbol{p}|$ measuring the in-plane spin projection onto the direction of momentum, the finite gap opening leads to the inequality $|\langle\hat{h}\rangle|<1$. This generates a spin tilting out of the plane.

For a closed boundary delineating two domains with gap openings (or masses) $\Delta_{1}$ (outside) and $\Delta_{2}$ (inside), depending on the relative sign and size of the gap (mass), distinct phases can be defined in the $\left(\Delta_{1}, \Delta_{2}\right)$ plane, as shown in Fig. 1(b). Among them, the quantum dot confinement phases, i.e., regions of $\left|\Delta_{1}\right|>\left|\Delta_{2}\right|$, are divided into two classes: inverted case with $\Delta_{1} \Delta_{2}<0$ [1 in Fig. 1(b)] and noninverted case with $\Delta_{1} \Delta_{2}>0$ [(2) in Fig. 1(b)]. In light of the JackiwRebbi paradigm and the associated index theorem that relates in-gap excitations to the topological charges of the defined 
background field $[48,49]$, robust in-gap modes are expected in the former case even without any magnetic field $(\boldsymbol{A}=0)$, but not in the latter case. Quite surprisingly, as sketched in Fig. 1(c) and demonstrated below, we find that the latter confinement configuration can bear such modes as well.

\section{A. Unconventional chiral edge states without band inversion}

For a circularly shaped dot geometry with the inner and outer regions defined by radius $R$, i.e., $\Delta(r)=\Delta_{1} \Theta(r-R)+$ $\Delta_{2} \Theta(R-r)$, the Dirac equation can be analytically solved in the polar coordinates $\boldsymbol{r}=(r, \theta)$, where the Hamiltonian is

$$
\hat{H}=\left(\begin{array}{ccc}
\Delta & \hat{D}_{-} & 0 \\
\hat{D}_{+} & 0 & \hat{D}_{-} \\
0 & \hat{D}_{+} & -\Delta
\end{array}\right),
$$

with

$$
\hat{D}_{ \pm}=\frac{\hbar}{\sqrt{2}} e^{ \pm i \theta}\left[-i v_{F}\left(\partial_{r} \pm \frac{i}{r} \partial_{\theta}\right)+\alpha_{ \pm}\right] .
$$

Under the symmetric gauge $\boldsymbol{B}=\boldsymbol{\nabla} \times \boldsymbol{A}=B / 2(-y, x)$, the magnetic field term is $\alpha_{ \pm}= \pm i \omega_{B} / 2 l_{B}$ with cyclotron frequency $\omega_{B}=v_{F} / l_{B}$ and magnetic length $l_{B}=\sqrt{\hbar / e B}$. Because the $z$ component of the total angular momentum $\hat{J}_{z}=-i \hbar \partial_{\theta}+\hbar \hat{S}_{z}$ commutes with $\hat{H}:\left[\hat{J}_{z}, \hat{H}\right]=0$, the common set of eigenstates takes the general form

$$
\Psi_{l}(\boldsymbol{r})=\left[\mathcal{R}_{1}(r) e^{i(j-1) \theta}, \mathcal{R}_{2}(r) e^{i j \theta}, \mathcal{R}_{3}(r) e^{i(j+1) \theta}\right]^{T}
$$

with the total angular momentum quantum number $j \in \mathbb{Z}$. For the dispersive bands, in the absence of any external magnetic field, we have

$$
\Psi_{j}^{\mu}(\boldsymbol{r})=\frac{C_{\mu}}{\sqrt{2}}\left(\begin{array}{c}
\alpha_{\mu} Z_{j-1}^{\mu}\left(k_{\mu} r\right) e^{-i \theta} \\
i \sqrt{2} Z_{j}^{\mu}\left(k_{\mu} r\right) \\
-\beta_{\mu} Z_{j+1}^{\mu}\left(k_{\mu} r\right) e^{i \theta}
\end{array}\right) e^{i j \theta},
$$

where the index $\mu=1,2$ labels the outer and inner regions of the circular domain boundary, $\alpha_{\mu}=\hbar v_{F} k_{\mu} /\left(E-\Delta_{\mu}\right)$, and $\beta_{\mu}=\hbar v_{F} k_{\mu} /\left(E+\Delta_{\mu}\right)$ with $\hbar v_{F} k_{\mu}=\sqrt{E^{2}-\Delta_{\mu}^{2}}, Z_{j}^{2}(x)=$ $J_{j}(x)$, and $Z_{j}^{1}(x)=H_{j}^{(1)}(x)$ are the Bessel and Hankel functions of the first kind, respectively. The eigenstates can be obtained by imposing continuity at the interface $R$, as shown in Appendix A.

Figure 2(a) shows the eigenenergies versus $j$. Given $\Delta_{1}=$ $36\left(\hbar v_{F} / R\right)$, for the conventional case of band inversion: $\Delta_{2}=-6\left(\hbar v_{F} / R\right)$, a branch of in-gap edge states arises [left panel of Fig. 2(a)], as expected. In addition, there are two other branches of edge modes located outside of the gap, which are reminiscent of the quantized, equatorial Rossby waves. The nonquantized version of these waves has been uncovered recently [50], which has stimulated an interest in the bulk-edge correspondence. Here, the waves represent a feature of quantization of spin-1 Dirac particles in the configuration of quantum-dot confinement. Surprisingly, for the noninverted case with $\Delta_{2}=+6\left(\hbar v_{F} / R\right)$, a class of unusual edge modes also emerges in the gap, as shown in the right panel of Fig. 2(a). Examining the associated spin projections onto the plane $\left(S_{\theta}, S_{z}\right)=\left(\left\langle v\left|\hat{\boldsymbol{S}} \cdot \hat{\boldsymbol{n}}_{\theta}\right| v\right\rangle,\left\langle v\left|\hat{\boldsymbol{S}}_{z}\right| v\right\rangle\right)$ with $\hat{\boldsymbol{n}}_{\theta}=$ $(\cos \theta, \sin \theta)$ and the current expectation value $u=\int \boldsymbol{u} \cdot d \boldsymbol{r}$ with $\boldsymbol{u}=\left\langle\nu\left|\nabla_{p} \hat{H}\right| v\right\rangle$ for eigenstates $|v\rangle$, as indicated by the arrows and scaled by colors, respectively, in Fig. 2(b), we see that the unexpected states exhibit the feature of unidirectional current-carrying similar to that of conventional topological states with band inversion. This coincides with the linear or monotonic dispersion relation between energy and angular momentum for in-gap excitations with its slope $d E_{j} / d j$ indicating the current direction. Remarkably, this phenomenon generally emerges in quantum dot confinement of noninverted type-I junctions, i.e., $\left|\Delta_{1}\right|>\left|\Delta_{2}\right|$ and $\Delta_{1} \Delta_{2}>0$. A concrete example of the dependence of the eigenenergy on the inner domain band gap $\Delta_{2}$ is presented in Fig. 2(d) with different markers denoting the corresponding states whose spatial density profiles are plotted in Fig. 2(c), for a fixed positive value of the outer domain band gap. We see that peculiar evolution of the bulk spectrum into chiral (unidirectional current-carrying) in-gap edge states occurs as $\Delta_{2}$ varies from the negative to the positive side in spite of the absence of band inversion. A quite remarkable phenomenon is the persistence of the chiral zero-energy modes with the characteristic feature of fully (in-plane) spin-current locking. The directions of the associated current and spin are simply determined by the sign of the band gap (or Dirac-type mass), which can be rigorously demonstrated in general (see Appendix A 2). Specifically, in the limit of hard-wall confinement $\Delta_{1} \rightarrow \infty$, the zero-energy modes have the form

$$
\Psi_{0}(\boldsymbol{r}) \sim\left(\begin{array}{c}
i I_{1}\left(\left|\delta_{2}\right| r\right) e^{-i \theta} \\
\operatorname{sign}\left(\delta_{2}\right) \sqrt{2} I_{0}\left(\left|\delta_{2}\right| r\right) \\
-i I_{1}\left(\left|\delta_{2}\right| r\right) e^{i \theta}
\end{array}\right) \Theta(R-r),
$$

rendering fully in-plane polarized the spatial spin texture $\boldsymbol{S}=\left[S_{x}, S_{y}, S_{z}\right] \sim \operatorname{sign}\left(\delta_{2}\right)[\sin \theta,-\cos \theta, 0]$, and leading to a vanishing (outward) normal current $u_{r}=\Psi_{0}^{\dagger} \hat{\boldsymbol{u}} \cdot \hat{\boldsymbol{n}}_{r} \Psi_{0}=0$ but a unidirectional azimuthal current

$$
u_{\theta}=\Psi_{0}^{\dagger} \hat{\boldsymbol{u}} \cdot \hat{\boldsymbol{n}}_{\theta} \Psi_{0} \approx-\operatorname{sign}\left(\delta_{2}\right) I_{0}\left(\left|\delta_{2}\right| r\right) I_{1}\left(\left|\delta_{2}\right| r\right) .
$$

As a result, for the emerging chiral zero-energy modes, we have $\boldsymbol{u} \cdot \boldsymbol{S} /|\boldsymbol{u}| \equiv 1$, signifying a perfect spin-current locking. Remarkably, these modes are not subject to any finite-size effect due to the confinement [51] and are analogous to the Majorana modes in topological superconductors because of the persistent vortex currents and the same type of symmetries obeyed [47].

\section{B. Robustness}

The appealing features of conventional topological chiral edge modes are zero dissipation and robustness against backscattering. Effectively, they execute one-way propagation along the boundary even in the presence of geometric deformations or impurities. A key question is whether the unconventional edge modes, due to lack of band inversion and bulkedge correspondence, have similar features. To address this question, we investigate the effects of geometric deformations in the domain boundary on these modes. For an arbitrarily shaped domain without the circular symmetry, to calculate the solutions of the Dirac equation for three-component spinors is extremely challenging. We have developed an accurate and efficient numerical method to find the solutions (Appendix B). 


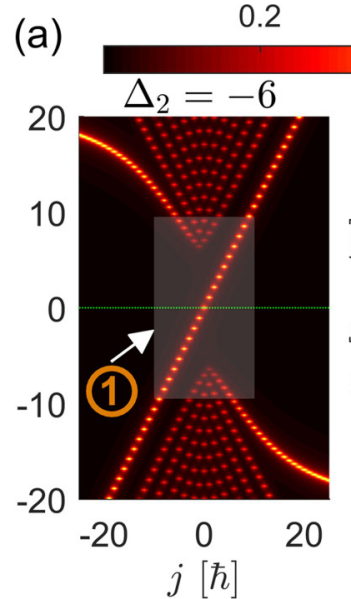

(b)

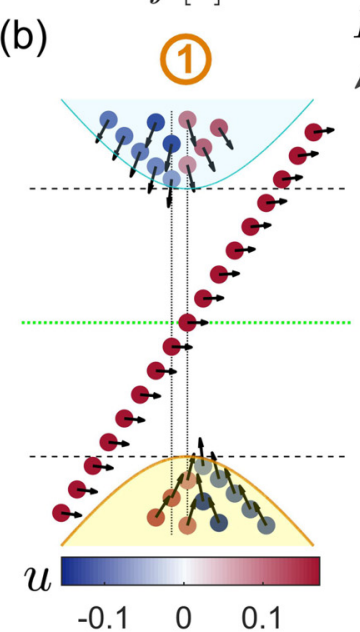

$0.4 \quad 0.6$

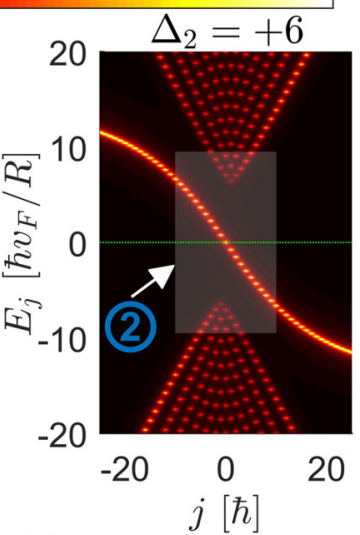

(2)

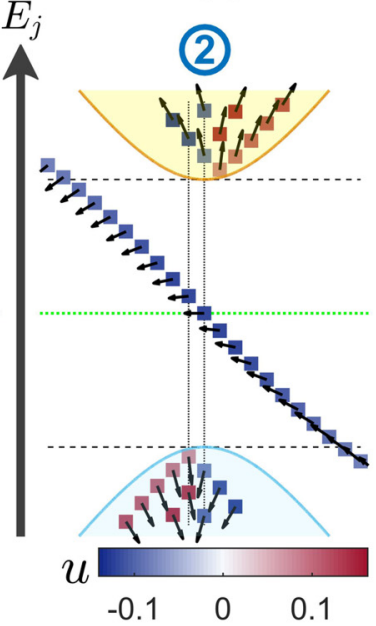

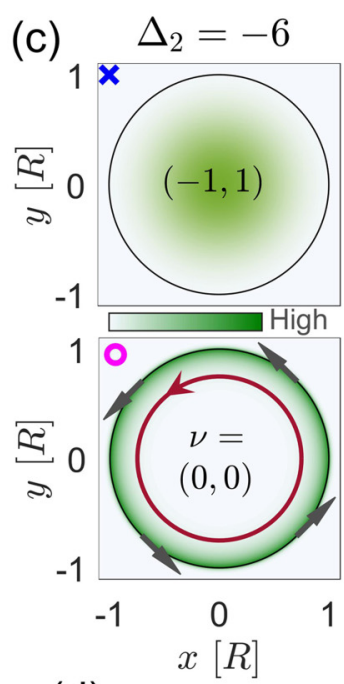
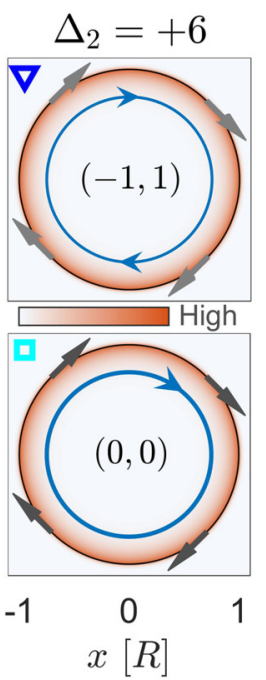

(d)

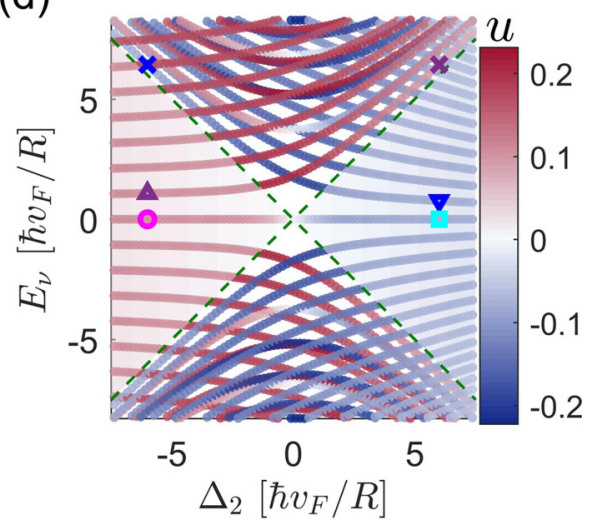

FIG. 2. Unique chiral edge states and spectrum evolution in spin-1 Dirac insulator quantum dots. (a) Eigenenergies versus total angular momentum for the conventional case of inverted band order/alignment characterized by a negative bulk band gap inside the dot $\Delta_{2}=-6$ $\left(\hbar v_{F} / R\right)$ (left), and the case without band inversion $\Delta_{2}=+6\left(\hbar v_{F} / R\right)($ right $)$, with the exterior bulk band gap (Dirac-type mass) fixed at $\Delta_{1}=$ $+36\left(\hbar v_{F} / R\right)$. Color bar shows the magnitude of the LDOS (local density of states) on a square-root scale. (b) Spectral features characterized by the circulating current $u$ with its values color coded and spin orientation denoted by the arrows in the $\left(S_{\theta}, S_{z}\right)$ plane. (c) Spatial density profile of representative states [indicated by different markers in (d)] with specific quantum numbers $v=(j, n):(0,0)$ for the zero-energy mode (bottom panels) and $(-1,1)$ for the first positive-energy mode of $j=1$ (top panels) for inverted (left) and noninverted cases (right). (d) Energy levels versus $\Delta_{2}$ with the given fixed value of $\Delta_{1}$.

Figure 3 shows some representative results, where the eigenspectra correspond to the resonance peaks in the dependence of the total density of states (DOS) on energy, as shown in Figs. 3(a) and 3(b) for the inverted and noninverted cases, respectively. We see that in-gap bound modes arise in both cases (the light red and blue shaded regions with $|E|<2$ ). For a family of geometric deformations characterized by the deformed strength parameter $d$, we find that such modes persist in the noninverted case and exhibit patterns similar to those of modes in systems with topological band inversion.

For a conventional topological insulator based quantum dot with band inversion, the edge states possess a large orbital magnetic moment $\mu_{o}=e / 2 \int \boldsymbol{r} \times \boldsymbol{u} d^{2} \boldsymbol{r}$, which can be exploited as an efficient probe to characterize topological protection against disorder [52,53]. We use the orbital magnetic moment to quantitatively characterize the chiral current-carrying property of the unconventional topological states and their robustness against geometric deformation. Figure 3(c) shows the calculated orbital moment $\mu_{0}$ for the in-gap bound modes arising from the inverted (red circles) and noninverted (blue squares) quantum dots, as well as the bounded bulk band states (gray diamonds) as a function of the geometric deformation parameter $d$. The results indicate that the unconventional chiral edge states without engaging any topological band inversion have a comparable level of robustness as the conventional topological states with band inversion. The feature of lack of backscattering, as revealed by the independence of $\mu_{o}$ on the deformation in Fig. 3(c), can also be seen from the spatial profiles of the corresponding states for a given deformed geometry, as displayed in Fig. 3(d).

\section{Spin-1 Fock-Darwin and Landau-level states}

To further ascertain the emergence of the edge states without band inversion, we study the Fock-Darwin characteristics of the states by solving the Dirac equation (Appendix A 3) in the presence of a magnetic field $\boldsymbol{B}$ under the symmetric gauge. 
(a)

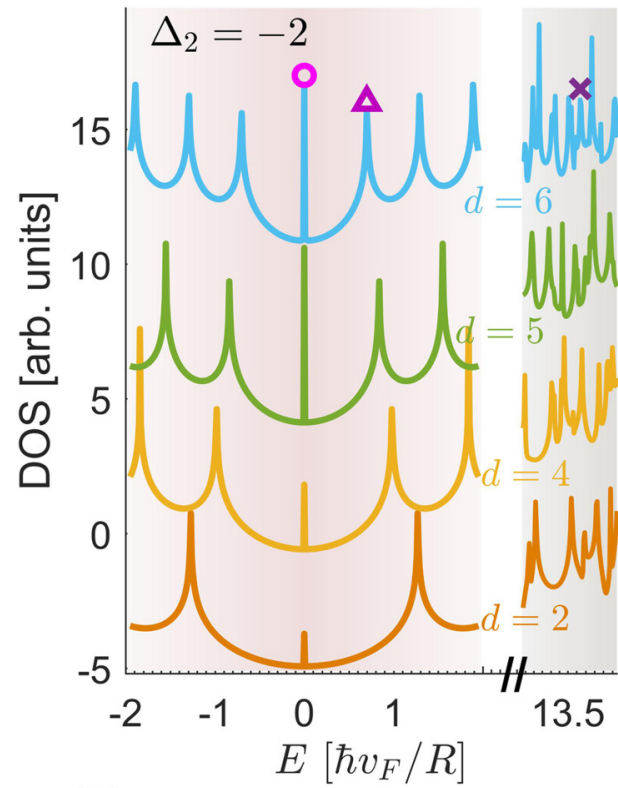

(b)

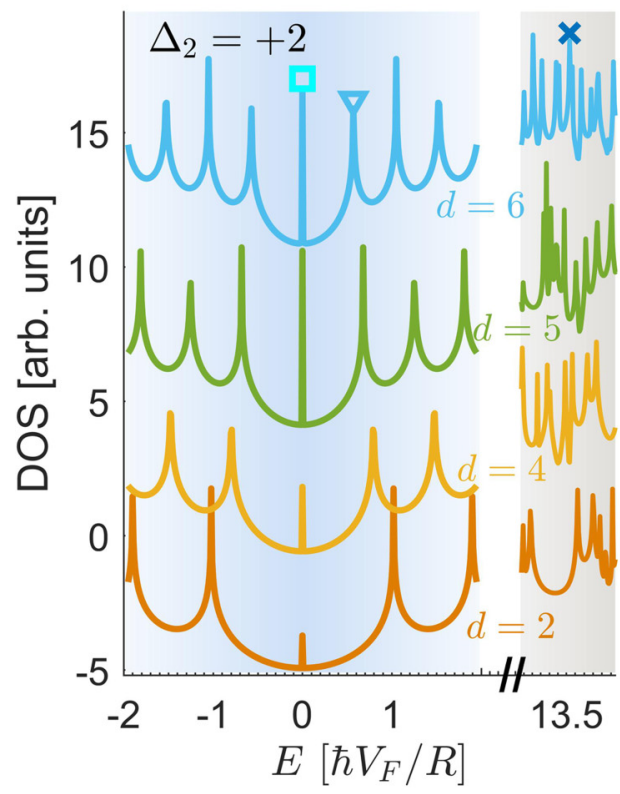

(c)

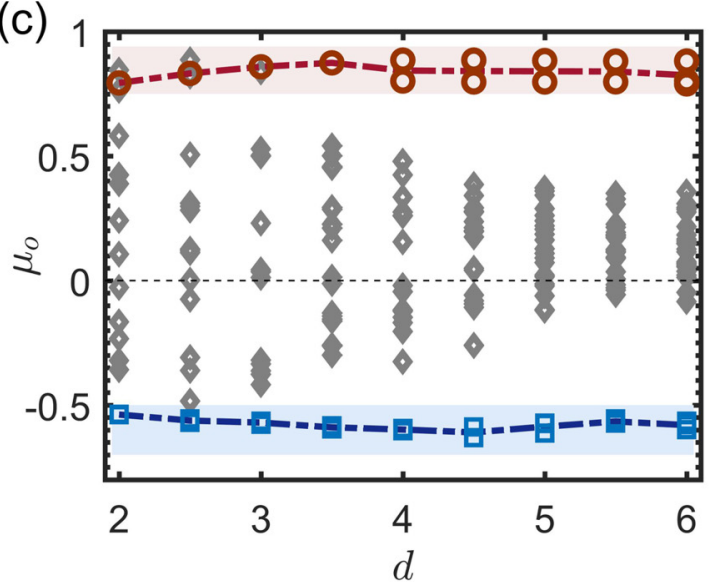

(d)

$\Delta_{2}=-2$

$\Delta_{2}=+2$

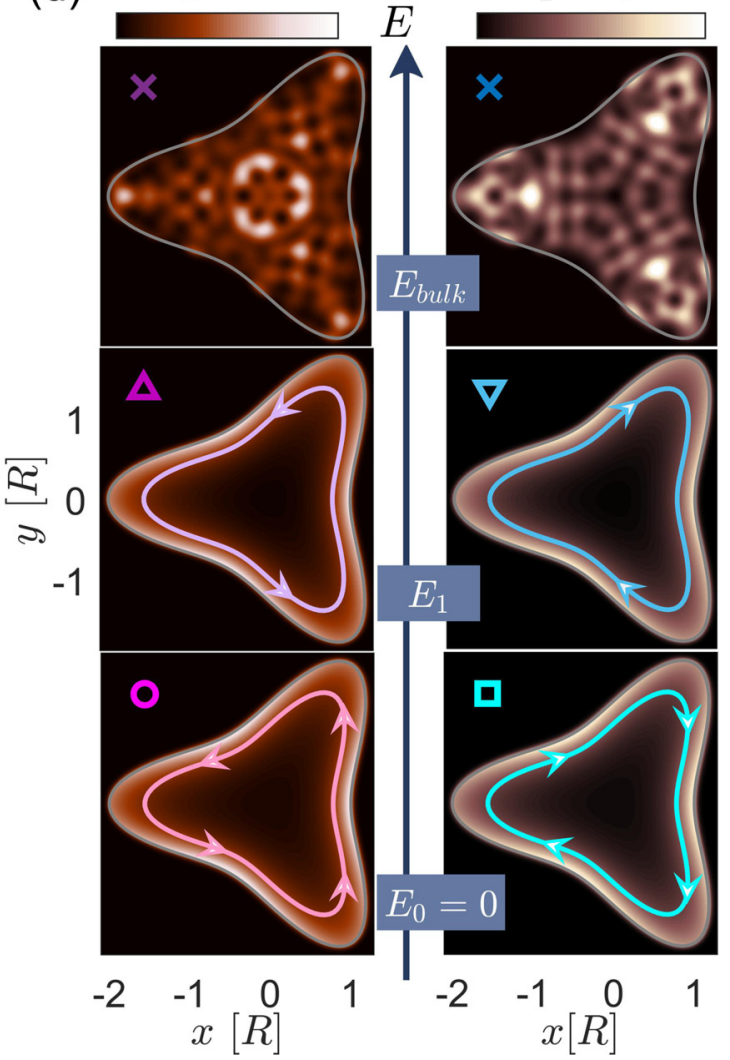

FIG. 3. Robustness comparison between conventional and unconventional topological chiral edge states against boundary/geometric deformation. For $\Delta_{1}=+20\left(\hbar v_{F} / R\right)$, DOS as a function of energy for cases with (a) inverted band order $\Delta_{2}=-2$ and (b) noninverted order $\Delta_{2}=+2$, where the peaks of each curve associated with a specific deformation parameter $d$ ( $d=2$ for the case of a circularly closed boundary and $d>2$ for irregular shaped boundaries) indicate the corresponding bound states. (Different curves are shifted vertically for better visualization). (c) Orbital moment $\mu_{o}$ versus $d$, where the red circles and blue squares are for the in-gap states obtained from (a) inverted and (b) noninverted cases with eigenenergies $\left|E_{n}\right|<\left|\Delta_{2}\right|$ (light red and light blue shaded areas), respectively. The gray diamonds correspond to the usual bounded bulk states obtained from (b) (gray shaded region with $E_{n}>13$ ). (d) Left column: real-space topography of densities $\left|\Psi_{n}\right|^{2}$ of the zeroth and the first positive-energy states in the gap as well as one representative ordinary state outside of the gap as indicated by different colored markers in (a). Right column: the corresponding case for the states marked in (b).

As for the case of conventional Fock-Darwin states under band inversion, for a weak magnetic field, quantum dot type of confinement is dominant. However, for a strong field, the vector potential term leads to highly degenerate Landau levels, as shown in Fig. 4. Remarkably, they are quite resistant against coalescence and generate a peculiar Fock-Darwin spectrum that signifies persistent paramagnetic currents $I_{j}=-\partial E_{j} / \partial B$. Because of the absence of gap inversion, the Landau-level type of confinement is normal. As a result, the interfacial states at a weak field are expelled from the gapped region as the Landau-level confinement becomes dominant. (A detailed analysis of the Landau levels is presented in Appendix A 3.) 
(a)

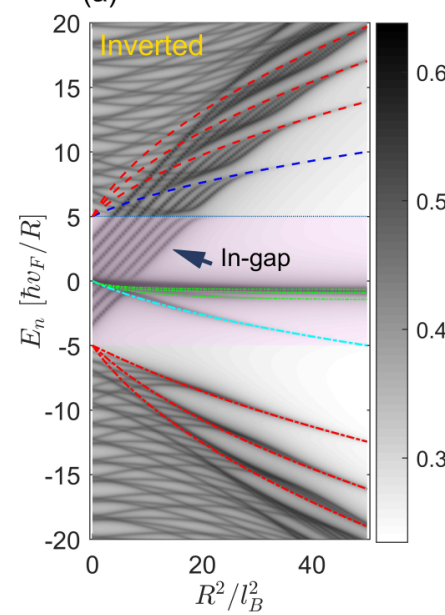

(b)

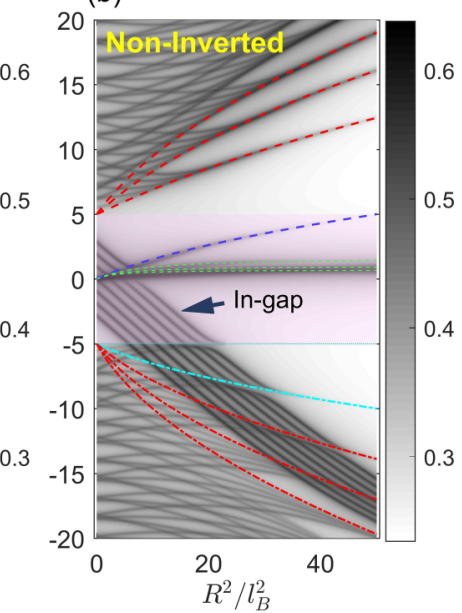

FIG. 4. Spin-1 Fock-Darwin states. Energy-level evolution as a function of the magnetic field parametrized by $R^{2} / l_{B}^{2}$, where $l_{B}=$ $\sqrt{\hbar / e B}$ is the magnetic length, for (a) $\Delta_{2}=-5$, a conventional case with band inversion, and (b) $\Delta=+5$, an unconventional case without band inversion, where we set $\Delta_{1}=+100\left(\hbar v_{F} / R\right)$ to guarantee quantum dot confinement. In both cases, the dashed curves denote the corresponding Landau levels.

\section{REGULARIZATIOIN ASSOCIATED WITH BULK TOPOLOGY AND BULK-EDGE CORRESPONDENCE}

\section{A. Anamalous chiral edge modes in noninverted, regularized, continuum spin-1 quantum dots}

We have demonstrated the emergence of chiral edge modes in noninverted spin-1 Dirac quantum dot systems based on the continuum model of generalized Dirac Hamiltonian subject to a confining potential of the massive type. The positiondependent, Dirac-type of mass profile can be viewed as an external perturbation/field associated with the real-space spin texture parametrized by $\hat{\boldsymbol{M}}(\boldsymbol{r})=\operatorname{sgn}(\Delta(\boldsymbol{r})) \hat{\boldsymbol{e}}_{z}$. The significance of our finding of the edge modes, which defy conventional wisdom, may be appreciated by drawing a comparison with the spin- $\frac{1}{2}$ counterpart system. In this regard, a paradigm in the study of spin- $\frac{1}{2}$ particle confinement is the JackiwRebbi model [6,7], in which the inverted configuration with sign-changing Dirac mass (i.e., $\Delta_{1} \Delta_{2}<0$ ) defines a realspace topological defect of domain wall characterized by a topological charge as

$$
\begin{aligned}
\mathcal{N} & =\frac{1}{4 \pi} \iint d^{2} \boldsymbol{r} \hat{\boldsymbol{M}} \cdot\left[\frac{\partial \hat{M}}{\partial x} \times \frac{\partial \hat{M}}{\partial y}\right] \\
& =\frac{1}{2}\left[\operatorname{sgn}\left(\Delta_{1}\right)-\operatorname{sgn}\left(\Delta_{2}\right)\right]= \pm 1,
\end{aligned}
$$

which leads to the in-gap chiral edge states at the dot boundary as expected (e.g., from the index theorem $[48,49]$ ). However, the noninverted configuration is topologically trivial and does not support such subgap modes. Our finding of the chiral edge modes in noninverted spin-1 Dirac quantum dot systems thus represents an exception that defies the conventional wisdom. Remarkably, the chiral edge modes arising from the topologically trivial confinement structure share all main characteristic features of in-gap chiral edge states such as spin-momentum locking, perfect unidirectionality, and the same degree of robustness as the modes engaged in the topological confinement of domain walls.

An established notion in understanding the topological wave phenomena is the bulk-edge correspondence that relates the net chiral edge modes at a boundary to the change in the relevant bulk topological invariants across it. A natural question is whether the anomalous chiral edge states can survive the "scrutiny" of the principle of bulk-edge correspondence. For spin- $\frac{1}{2}$ Dirac Hamiltonian, this issue was addressed earlier [54]. More recently, the issue has been studied in the context of topological waves in fluids [55]. Here, to study the bulk-edge correspondence for spin-1 Dirac particles, we construct a regularized continuum model of massive spin-1 particles with an additional quadratically dispersive mass term in momentum. The Hamiltonian is

$$
\mathcal{H}_{r}^{(1)}(\boldsymbol{k})=\left(k_{x}, k_{y}, f-\epsilon k^{2}\right) \cdot\left(\hat{S}_{x}, \hat{S}_{y}, \hat{S}_{z}\right) .
$$

With the regularization, the Hamiltonian is well behaved at the momentum infinity $|\boldsymbol{k}| \rightarrow \infty$, leading to well-defined topological invariants associated with all three bulk bands in the momentum space that are either trivial or nontrivial. The invariants are explicitly determined by the first Chern numbers given by

$$
\mathcal{C}_{ \pm}=\operatorname{sgn}(f)+\operatorname{sgn}(\epsilon)
$$

for the dispersive positive and negative bands, and $\mathcal{C}_{0}=0$ for the middle flat band [56]. The sign of the mass $f$ effectively plays the consequential role in identifying the bulk topology with given $\epsilon \neq 0$, despite that the quantity itself is not a Chern number. Modeling the quantum dot confinement of spin-1 particles through a position-dependent mass profile given by

$$
f(\boldsymbol{r})=f_{1} \Theta(|\boldsymbol{r}|-R)+f_{2} \Theta(R-|\boldsymbol{r}|),
$$

we conclude that the inverted case with $f_{1} f_{2}<0$ is associated with a discontinuous change in the bulk topological invariant (Chern number) across the dot boundary, while the noninverted one with $f_{1} f_{2}>0$ has a vanishing change and thus represents a topologically trivial boundary from the point of view of bulk-edge correspondence. For representative parameter values, we numerically solve the system and obtain the eigenspectra for each case with an irregular boundary shape, as shown in Figs. 5(a) and 5(b). We see that, conventionally unexpected in-gap edge modes can emerge in the noninverted case. This provides further confirmation of our finding of these topological modes in regularized systems in which the underlying bulk topology can be properly defined.

To present a stark contrast of the result in Fig. 5(b) to the conventional understanding, we solve the spin- $-\frac{1}{2}$ counterpart of the quantum dot system described by the following regularized two-band Dirac Hamiltonian:

$$
\mathcal{H}_{r}^{(1 / 2)}(\boldsymbol{k})=\left(k_{x}, k_{y}, f-\epsilon k^{2}\right) \cdot\left(\hat{\sigma}_{x}, \hat{\sigma}_{y}, \hat{\sigma}_{z}\right),
$$

with the corresponding results for the same set of parameter values shown in Figs. 5(c) and 5(d) for the inverted and noninverted configurations, respectively. As expected, for the spin- $\frac{1}{2}$ system, the in-gap boundary modes completely disappear in the case of noninverted quantum dot with the trivial bulk topology. 

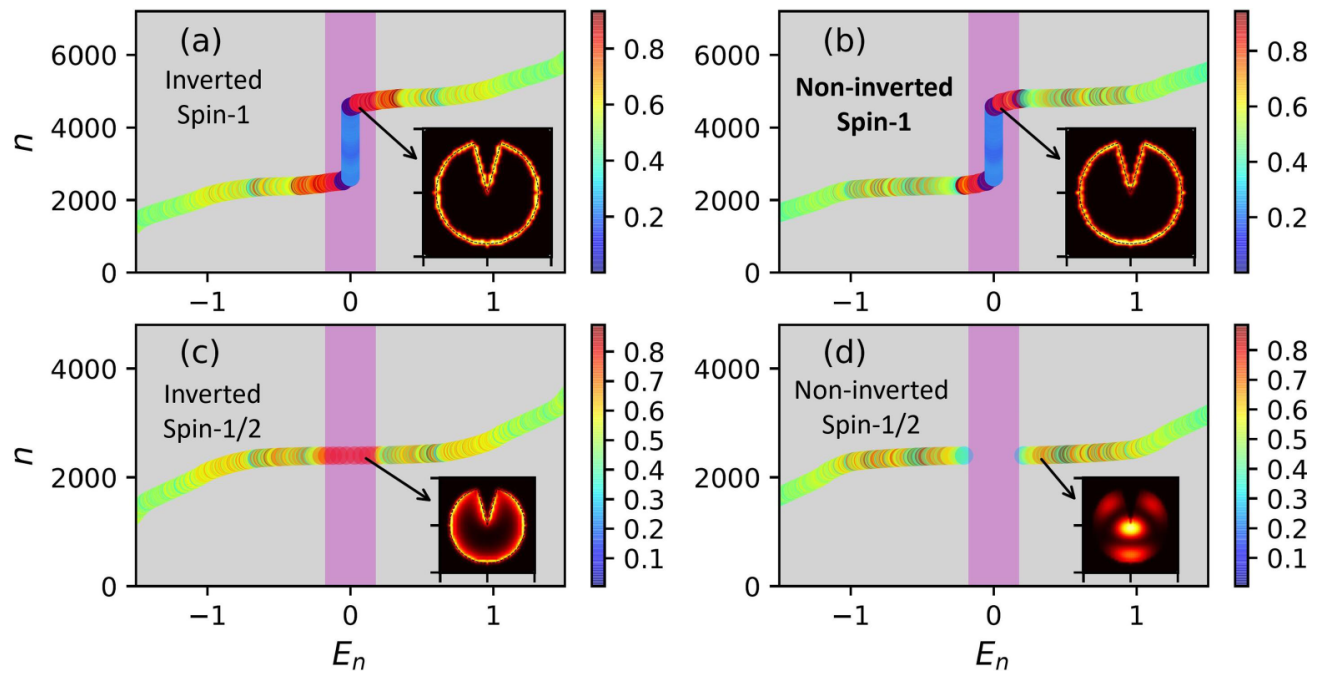

FIG. 5. Energy spectra of regularized continuum model of spin-1 and spin- $\frac{1}{2}$ Dirac quantum dots. The regularization parameter is $\epsilon=-0.2$. (a), (b) State number versus eigenenergies for the inverted and noninverted configurations of spin-1 Dirac particles, respectively, and (c), (d) the corresponding results for the spin- $\frac{1}{2}$ Dirac system. The values of other parameters are $\left(f_{1}=1, f_{2}=-\frac{1}{6}\right)$ and $\left(f_{1}=1, f_{2}=+\frac{1}{6}\right)$ for the inverted and noninverted configurations, respectively. The color bar indicates the LDOS intensity evaluated about the dot boundary.

\section{B. Anamalous chiral edge modes in noninverted lattice spin-1 quantum dots}

We demonstrate the emergence of the anomalous chiral edge modes in noninverted quantum dots using the experimentally accessible lattice systems. Particularly, the spin-1 generalization of the ordinary Dirac Hamiltonian can effec- tively describe relativisticlike, low-energy excitations of various lattice-based electronic and photonic materials $[17,31,36]$, where the regularization issue is inherently taken into account by the underlying Brillouin zone, a built-in compact torus manifold. In analogy to graphene [57], we consider the dicelattice-based realization of spin-1 systems, as illustrated in
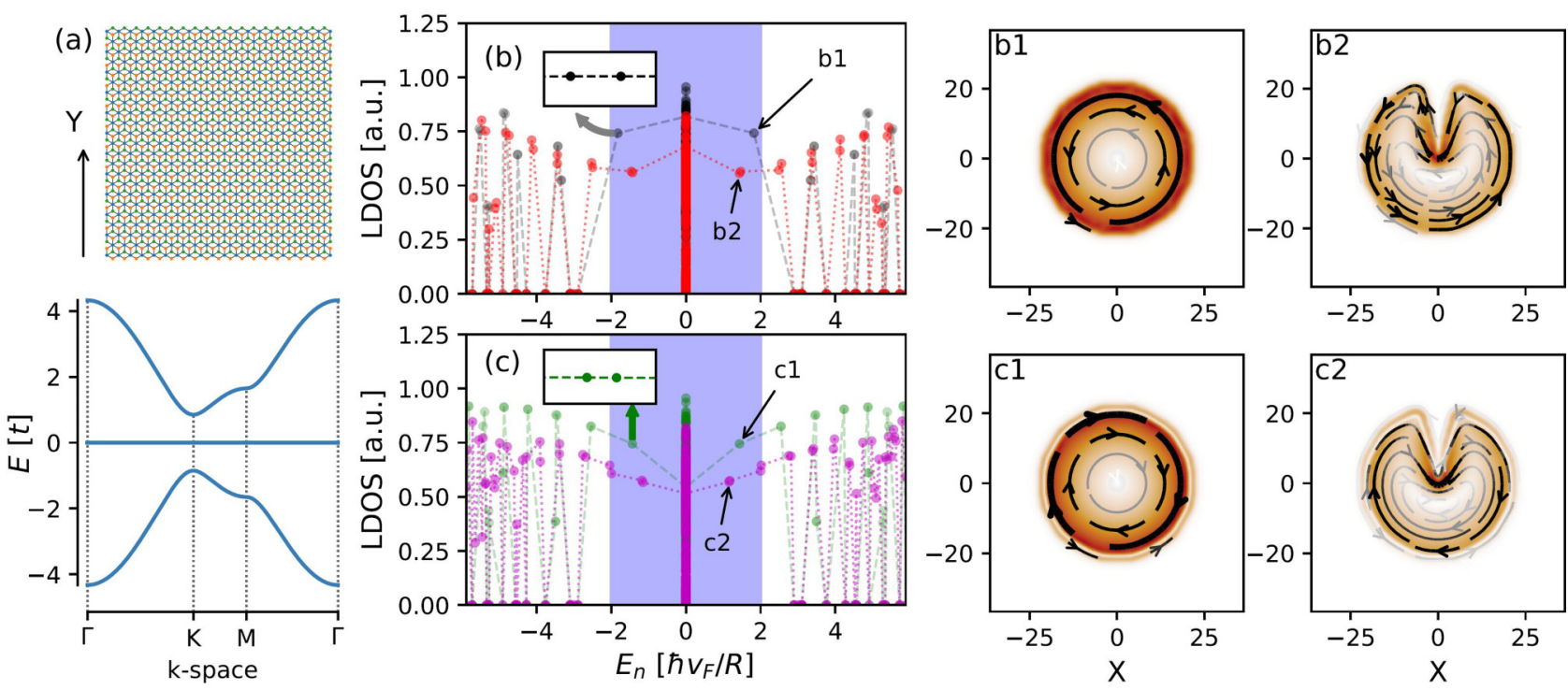

FIG. 6. Emergence of predicted anomalous chiral edge states in dice lattice systems/materials. (a) A schematic diagram of dice lattice with the Bravais lattice constant $a$ and nearest-neighbor hopping energy $t$ (top panel), and the associated bulk energy-momentum dispersion relation in the presence of a staggered sublattice potential $(\Delta / t=0.85)$, which is responsible for gap opening and the related bulk topology. Near the $K$ point, the massive spin-1 Dirac lattice system features low-energy bulk quasiexcitations in the long-wavelength regime. (b), (c) Dependence of LDOS on the eigenenergies for the inverted and noninverted, corresponding to the confinement configuration with parameter settings $\left(\delta_{1}, \delta_{2}\right)=(+20,-2)$ and $(+20,+2)$, respectively. These parameter values are adopted from the continuum spin-1 Dirac Hamiltonian based system for a meaningful comparison. The gray and green curves are for the circularly shaped quantum dot geometry, while the red and magenta are for the deformed dot. Insets are magnification of of the specified regions of the plots reflecting the two valley degrees of freedom. Panels (b1) and (b2) and (c1) and (c2) display the real-space distributions of the local current of the in-gap states belonging to one of the valleys as indicated in (b) and (c), respectively. 

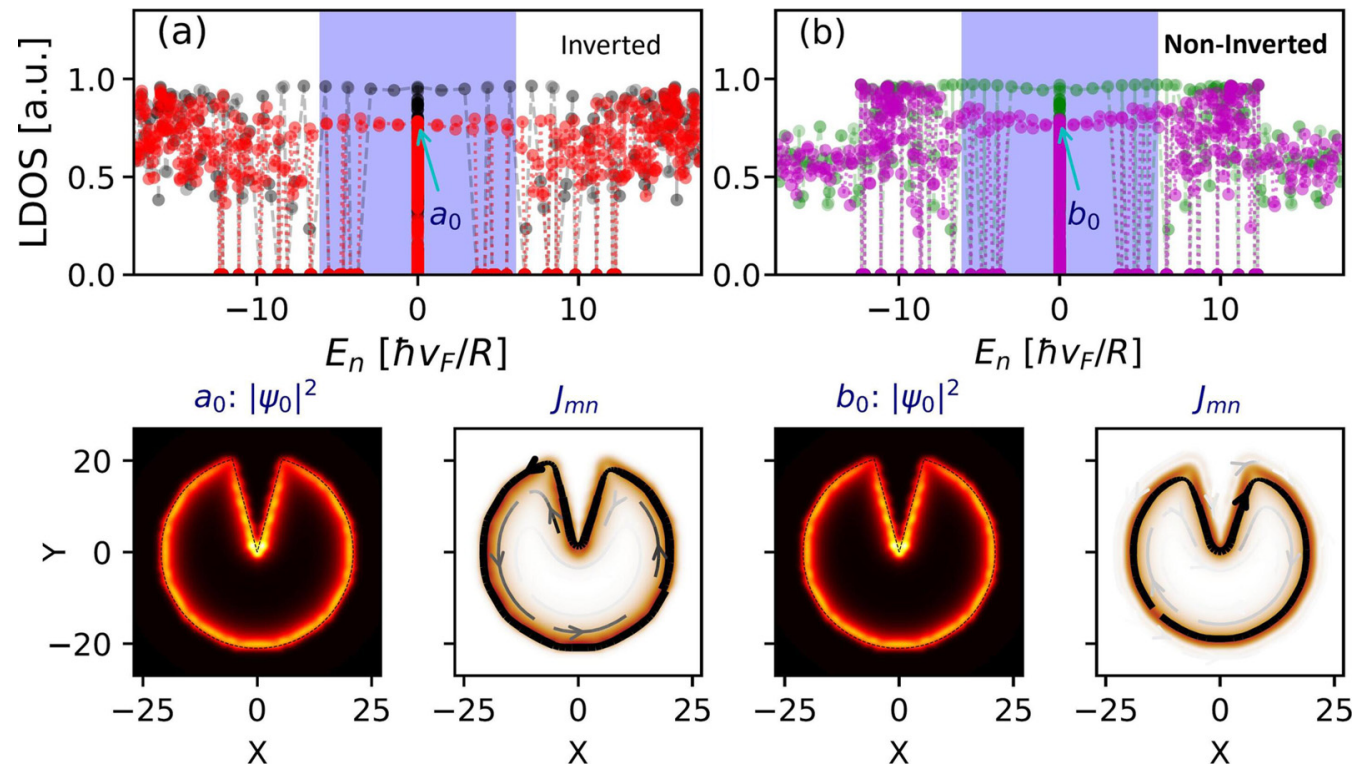

FIG. 7. Emergence of predicted anomalous chiral edge states in dice lattice for another parameter setting. The parameters are adopted from the simplified effective spin-1 Hamiltonian leading to the key results shown in Fig. 2. (a), (b) Dependence of LDOS on the eigenenergies for the inverted case $\left(\Delta_{2}=+6 \hbar v_{F} / R\right)$ and noninverted case $\left(\Delta_{2}=-6 \hbar v_{F} / R\right)$, respectively, for $\Delta_{1}=+36 \hbar v_{F} / R$. The zero modes obtained for both cases are indicated in panels $a_{0}$ and $b_{0}$. The corresponding real-space density $\left(\left|\psi_{0}\right|^{2}\right)$ and the current $\left(J_{m n}\right)$ topographies are displayed in the bottom panels.

Fig. 6(a). The tight-binding Hamiltonian in real space is

$$
\begin{aligned}
H_{\text {dice }}= & -t \sum_{\langle i, j\rangle}\left(c_{B i}^{\dagger} c_{A j}+c_{B i}^{\dagger} c_{C j}+\text { H.c. }\right) \\
& +\Delta \sum_{i}\left(c_{C i}^{\dagger} c_{C i}-c_{A i}^{\dagger} c_{A i}\right),
\end{aligned}
$$

where $c_{v i}^{\dagger}\left(c_{v i}\right)$ with $v=A, B, C$ being the creation (annihilation) operators of the localized states $|v i\rangle$ at site $i$ belonging to the sublattice $v$, and $\langle i, j\rangle$ denotes pairs of nearest-neighbor sites with the tunneling strength (hopping energy) $t \approx 3 \mathrm{eV}$. The last term represents a staggered sublattice potential responsible for the Dirac type of mass-based gap opening. A position-dependent staggered potential is applied to implement the two cases of quantum dot confinement: inverted and noninverted, as in the effective continuum model. The resulting spectral properties are predicted and characterized by the LDOS evaluated about the dot boundary so as to probe the edge states effectively. Given the Bravais lattice constant $a=0.246 \mathrm{~nm}$, we set the typical size of the dot to be $21 a \sim$ $5 \mathrm{~nm}$, contained within a larger square flake of edge size of $70 a \sim 17 \mathrm{~nm}$, so the eigenspectra and the edge states in the quantum dot confinement can be calculated. We use the open source KWANT package [58] to perform the calculations.

Figures 6(b), 6(b1), 6(b2) and 6(c), 6(c1), 6(c2) show the representative results. We see that the tight-binding latticemodel-based simulations reveal the same remarkable phenomenon as that predicted by the continuum model (without any regularization in defining a proper bulk topology, Fig. 2), where the in-gap edge states emerged in the topologically trivial case of the noninverted configuration are immune to relatively large geometric deformations as the topologically protected modes in the inverted case. Figure 7 shows the corresponding results but for a different confinement parameter set $\left(\Delta_{1}, \Delta_{2}\right)$ as specified in the corresponding continuum model [cf., Figs. 2 and 3]. As shown in the bottom panels of Fig. 7, highly polarized in-plane pseudospin edge currents and the real-space distributions of the probability density of the zero modes are visually indistinguishable between the noninverted and inverted cases, providing further confirmation of our theoretically predicted anomalous chiral edge states in the tight-binding lattice setting that is directly experimentally relevant. In both Figs. 6 and 7, the local current flowing from site $m$ to site $n$ is calculated by

$$
J_{m n}=\frac{i}{\hbar}\left[t_{m n} \psi_{m}^{\dagger} \psi_{n}-t_{n m} \psi_{m} \psi_{n}^{\dagger}\right]
$$

with $t_{m n}$ denoting the hopping amplitude between the two sites.

We note that the staggered sublattice potential breaks the inversion symmetry but preserves the time-reversal symmetry due to the presence of two nonequivalent valleys $K$ and $K^{\prime}$. As in the case of graphene [57], the two valleys carry opposite bulk topological charges $\mathcal{N}_{K}=-\mathcal{N}_{K^{\prime}}=\operatorname{sgn}(\Delta)$ (except the flat band), while the Chern number based bulk topological invariant always vanishes. As such, provided that intervalley couplings are negligible, the in-gap excitations constitute pairs of nearly degenerate modes circulating in opposite directions in the two valleys. This feature has in fact been captured in our simulated results as illustrated in the insets of Figs. 6(b) and 6(c). The physical significance is that the tight-binding Dice lattice implementation of the spin-1 Dirac quantum dot hosts two copies of the chiral edge states that can be thought of as "valley-helical" states, analogous to the spin-helical states in quantum spin Hall systems. Distinctively, our results suggest an unconventional but physical way to attain such states with appealing topological properties but without engaging the proper topological restriction as imposed by the conventional 
rules of either bulk-edge correspondence or the index theorem (which is relatively difficult to realize in experiments). Taken together, our finding opens a new avenue to realizing topologically protected, chiral edge states in 2D Dirac materials.

\section{CONCLUSION AND DISCUSSION}

Our study of spin-1 Dirac particles in a confining potential of the massive type, which belong to the same class-D symmetry (particle-hole symmetry $\mathcal{P}$ and broken time-reversal symmetry $\mathcal{T}$ ) as in two-dimensional $p+i p$ superconductors, has led to the discovery of a class of unconventional, backscattering-immune, chiral edge modes without engaging the topological restriction of band inversion. The uncovered mid-gap zero modes are reminiscent of the chiral Majorana modes in terms of the associated persistent vortex currents and the symmetry class obeyed. In the presence of an external magnetic field, the modes lead to a unique Fock-Darwin spectrum that endows them with experimentally detectable signatures of sizable persistent (paramagnetic) currents and magnetic moments. We expect the unconventional states to be experimentally realizable because they were unveiled under the same setting of quantum dot confinement as for the conventional topological states. The significance of our finding is that topologically protected states can arise in condensed matter systems even without topological restriction, opening a wider avenue for applications of topological quantum states. Our finding is also fundamental to the field of relativistic quantum wave dynamics.

To address the fundamental issue of regularization of spin1 Dirac Hamiltonian in the emergence of chiral edge states, we have constructed a class of regularized continuum models. Similar models have been used recently in classical fluids and plasma physics $[50,55,56]$ to study topological wave phenomena and the bulk-edge correspondence in classical continuum media. However, unlike all previous studies that focused on the well-defined nonvanishing bulk topology regime, our efforts have concentrated on searching for and understanding possible topological edge states in a topologically trivial setting in which such states were deemed impossible by the conventional wisdom. We have accomplished this goal by theoretically predicting the emergence of in-gap chiral edge states in spin-1 Dirac quantum dot systems, regardless of whether the system has or has no regularization, and regardless of whether the system is continuum or of the directly experimentally relevant lattice type. Remarkably, the anomalous in-gap chiral edge modes uncovered in our work can possess the characteristic traits of spin-current (momentum) locking, perfect unidirectionality, and the same level of robustness as those requiring the proper topological restriction with the zero modes. Our finding thus represents a contribution to the fundaments of the field of topological quantum states.

\section{ACKNOWLEDGMENTS}

We would like to acknowledge support from the Vannevar Bush Faculty Fellowship program sponsored by the Basic Research Office of the Assistant Secretary of Defense for Research and Engineering and funded by the Office of Naval Research through Grant No. N00014-16-1-2828.

\section{APPENDIX A: PROPERTIES OF GAPPED DIRAC MATERIALS AND SOLUTIONS OF THE GENERALIZED DIRAC-WEYL EQUATION}

\section{Basics}

We consider gapped Dirac materials hosting spin-1 lowenergy excitations. The governing equation is the generalized Dirac-Weyl equation for three-component spinor $\Psi(\boldsymbol{r})=$ $\left[\psi_{1}(\boldsymbol{r}), \psi_{2}(\boldsymbol{r}), \psi_{3}(\boldsymbol{r})\right]^{T}$ :

$$
\hat{H} \Psi(\boldsymbol{r})=\left[v_{F} \hat{\boldsymbol{S}} \cdot \hat{\boldsymbol{D}}+\Delta \hat{S}_{z}\right] \Psi(\boldsymbol{r})=E \Psi(\boldsymbol{r}),
$$

where $\hat{\boldsymbol{D}}=\hat{\boldsymbol{p}}+e \boldsymbol{A}$ with $\hat{\boldsymbol{p}}$ being the momentum operator is a scalar type of perturbation, e.g., an electrostatic potential. The local current $\hat{\boldsymbol{u}}$ associated with state $\Psi(\boldsymbol{r})$ can be calculated from the local expectation value as

$$
\begin{aligned}
\boldsymbol{u}(\boldsymbol{r}) & =v_{F}\left(\psi_{1}^{*}, \psi_{2}^{*}, \psi_{3}^{*}\right) \hat{\boldsymbol{S}}\left(\begin{array}{l}
\psi_{1} \\
\psi_{2} \\
\psi_{3}
\end{array}\right) \\
& =\sqrt{2} v_{F}\left(\operatorname{Re}\left[\psi_{2}^{*}\left(\psi_{1}+\psi_{3}\right)\right],-\operatorname{Im}\left[\psi_{2}^{*}\left(\psi_{1}-\psi_{3}\right)\right]\right) .
\end{aligned}
$$

By definition, the local current is the local probability density of spin vector $\left(S_{x}, S_{y}\right)$.

The Hamiltonian in Eq. (A1) possesses a particular type of discrete symmetry under the action of the antiunitary operators of time reversal $\mathcal{T}$ and particle-hole conjugate $\mathcal{P}$ defined as

$$
\begin{aligned}
& \mathcal{T}=\left.\left(\begin{array}{ccc}
0 & 0 & -1 \\
0 & 1 & 0 \\
-1 & 0 & 0
\end{array}\right) \mathcal{K}\right|_{k \rightarrow-k}, \\
& \mathcal{P}=\left.\left(\begin{array}{lll}
0 & 0 & 1 \\
0 & 1 & 0 \\
1 & 0 & 0
\end{array}\right) \mathcal{K}\right|_{k \rightarrow-k},
\end{aligned}
$$

where $\mathcal{K}$ is the operator for complex conjugation. In general, a finite gap opening perturbation, i.e., the Dirac-type mass $\Delta \neq 0$, will break the time-reversal symmetry $\mathcal{T} \hat{H} \mathcal{T}^{-1} \neq \hat{H}$ even without any externally applied magnetic field (i.e., $\boldsymbol{A}=0)$. However, the particle-hole symmetry is preserved: $\mathcal{P} H(\hat{\boldsymbol{p}}) \mathcal{P}^{-1}=-\hat{H}(-\hat{\boldsymbol{p}})$. We note that, in terms of symmetry classification, the spin-1 generalization of a conventional $(2+1)$-dimensional massive Dirac fermion Hamiltonian belongs to class $\mathrm{D}$ (with $\mathcal{P}^{2}=1$ and broken $\mathcal{T}$ ), which is the same as that of the Bogoliubov-de Gennes (BdG) Hamiltonian for the rare, $p+i p$ type of superconductors hosting chiral/unpaired Majorana modes [47,59].

In the gapped phase with a finite constant $\Delta$ and absence of external magnetic field $(\boldsymbol{A}=0)$, the eigenenergies of $\hat{H}$ are $E_{0}=0$ and $E_{ \pm}= \pm \sqrt{\Delta^{2}+\hbar v_{F}|\boldsymbol{k}|^{2}}$ with the wave vector $\boldsymbol{k}=\left(k_{x}, k_{y}\right)$ making an angle $\theta=\arctan \left(k_{y} / k_{x}\right)$ with the $x$ axis. The corresponding eigenvectors are

$$
|\boldsymbol{k}, 0\rangle=\frac{1}{\sqrt{2} \sqrt{|\boldsymbol{k}|^{2}+\delta^{2}}}\left[\begin{array}{lll}
k e^{-i \theta}, & -\sqrt{2} \delta, & -k e^{i \theta}
\end{array}\right]^{T}
$$

and

$$
|\boldsymbol{k}, s\rangle=\frac{\sqrt{\epsilon_{s}^{2}-\delta^{2}}}{2\left|\epsilon_{s}\right|}\left(\begin{array}{c}
\alpha e^{-i \theta} \\
\sqrt{2} \\
\beta e^{i \theta}
\end{array}\right),
$$

where $\alpha=\sqrt{\epsilon_{s}^{2}-\delta^{2}} /\left(\epsilon_{s}-\delta\right)$ and $\beta=\sqrt{\epsilon_{s}^{2}-\delta^{2}} /\left(\epsilon_{s}+\delta\right)$ with $\delta=\Delta / \hbar v_{F}, \epsilon_{s}=E_{s} / \hbar v_{F}$, and $s= \pm$. 
In the polar coordinates $\boldsymbol{r}=(r, \theta)$, Eq. (A1) becomes

$$
\left(\begin{array}{ccc}
\delta & \hat{\boldsymbol{D}}_{-} & 0 \\
\hat{\boldsymbol{D}}_{+} & 0 & \hat{\boldsymbol{D}}_{-} \\
0 & \hat{\boldsymbol{D}}_{+} & -\delta
\end{array}\right)\left(\begin{array}{l}
\psi_{1} \\
\psi_{2} \\
\psi_{3}
\end{array}\right)=\epsilon\left(\begin{array}{l}
\psi_{1} \\
\psi_{2} \\
\psi_{3}
\end{array}\right),
$$

where

$$
\hat{\boldsymbol{D}}_{ \pm}=-\frac{i}{\sqrt{2}} e^{ \pm i \theta}\left[\left(\partial_{r} \pm \frac{i}{r} \partial_{\theta}\right)+i a_{ \pm}\right]
$$

with

$$
\begin{aligned}
a_{ \pm} & =\left(\hat{\boldsymbol{e}}_{r} \pm i \hat{\boldsymbol{e}}_{\theta}\right) \cdot \boldsymbol{A} e / \hbar, \\
\hat{\boldsymbol{e}}_{r} & =(\cos \theta, \sin \theta), \quad \hat{\boldsymbol{e}}_{\theta}=(-\sin \theta, \cos \theta) .
\end{aligned}
$$

\section{Eigensolutions of dot confinement without magnetic field}

Analytical solutions. We consider a circularly shaped nonuniform gap opening profile that describes a quantum dot confinement geometry of radius $R$ :

$$
\Delta(r)=\Delta_{\mathrm{I}} \Theta(r-R)+\Delta_{\mathrm{II}} \Theta(R-r) .
$$

For $\boldsymbol{A}=0$, the common set of eigenstates has the general form

$$
\Psi_{l}(\boldsymbol{r})=\left[\mathcal{R}_{1}(r) e^{i(l-1) \theta}, \mathcal{R}_{2}(r) e^{i l \theta}, \mathcal{R}_{3}(r) e^{i(l+1) \theta}\right]^{T},
$$

where $l \in \mathbb{Z}$ is the total angular momentum quantum number. The associated local current density is $\boldsymbol{u}(r, \theta)=u_{r} \hat{\boldsymbol{e}}_{r}+u_{\theta} \hat{\boldsymbol{e}}_{\theta}$ with

$$
\begin{aligned}
& u_{\theta}=-\sqrt{2} v_{F} \operatorname{Im}\left[\mathcal{R}_{2}^{*}\left(\mathcal{R}_{1}-\mathcal{R}_{3}\right)\right], \\
& u_{r}=\sqrt{2} v_{F} \operatorname{Re}\left[\mathcal{R}_{2}^{*}\left(\mathcal{R}_{1}+\mathcal{R}_{3}\right)\right] .
\end{aligned}
$$

For the two dispersive bands, we have

$$
\Psi_{l}^{\mu}(\boldsymbol{r})=\frac{C_{\mu}}{\sqrt{2}}\left(\begin{array}{c}
\alpha_{\mu} Z_{l-1}^{\mu}\left(k_{\mu} r\right) e^{-i \theta} \\
i \sqrt{2} Z_{l}^{\mu}\left(k_{\mu} r\right) \\
-\beta_{\mu} Z_{l+1}^{\mu}\left(k_{\mu} r\right)^{i \theta}
\end{array}\right) e^{i l \theta},
$$

where the indices $\mu=\mathrm{I}$, II label the outer and inner regions of the circular domain boundary, respectively, and the parameters are

$$
\begin{aligned}
\alpha_{\mu} & =\hbar v_{F} k_{\mu} /\left(E-\Delta_{\mu}\right), \\
\beta_{\mu} & =\hbar v_{F} k_{\mu} /\left(E+\Delta_{\mu}\right), \\
\hbar v_{F} k_{\mu} & =\sqrt{E^{2}-\Delta_{\mu}^{2}},
\end{aligned}
$$

and $Z_{l}^{\mathrm{II}}(x)=J_{l}(x)$ and $Z_{l}^{\mathrm{I}}(x)=H_{l}^{(1)}(x)$ are Bessel and Hankel functions of the first kind, respectively. Matching the spinor wave functions $\Psi_{l}^{\mathrm{II}}$ and $\Psi_{l}^{\mathrm{I}}$ at the domain boundary (interface) $r=R$, we obtain the following transcendental equation:

$$
\begin{gathered}
J_{l}\left(k_{\mathrm{II}} R\right)\left[\frac{\sqrt{E^{2}-\Delta_{\mathrm{I}}^{2}}}{E-\Delta_{\mathrm{I}}} H_{l-1}^{(1)}\left(k_{\mathrm{I}} R\right)-\frac{\sqrt{E^{2}-\Delta_{\mathrm{I}}^{2}}}{E+\Delta_{\mathrm{I}}} H_{l+1}^{(1)}\left(k_{\mathrm{I}} R\right)\right] \\
=H_{l}^{(1)}\left(k_{\mathrm{I}} R\right)\left[\frac{\sqrt{E^{2}-\Delta_{\mathrm{II}}^{2}}}{E-\Delta_{\mathrm{II}}} J_{l-1}\left(k_{\mathrm{II}} R\right)\right. \\
\left.-\frac{\sqrt{E^{2}-\Delta_{\mathrm{II}}^{2}}}{E+\Delta_{\mathrm{II}}} J_{l+1}\left(k_{\mathrm{II}} R\right)\right]
\end{gathered}
$$

which can be calculated numerically to yield the eigenenergies and eigenstates with high accuracy. In particular, for an energy value within the bulk band gap, i.e., $|E|<\left|\Delta_{\mathrm{I} / \mathrm{II}}\right|$, the radial wave numbers are purely imaginary and can be redefined as

$$
\begin{aligned}
& k_{\mathrm{I}} R=\frac{\sqrt{E^{2}-\Delta_{\mathrm{I}}^{2}}}{\hbar v_{F} / R}=\sqrt{\epsilon^{2}-\delta_{\mathrm{I}}^{2}}=i \kappa_{\mathrm{I}} ; \\
& k_{\mathrm{II}} R=\frac{\sqrt{E^{2}-\Delta_{\mathrm{II}}^{2}}}{\hbar v_{F} / R}=\sqrt{\epsilon^{2}-\delta_{\mathrm{II}}^{2}}=i \kappa_{\mathrm{II}} .
\end{aligned}
$$

With the substitutions

$$
K_{l}(x)=\frac{\pi}{2} i^{l+1} H_{l}^{(1)}(i x), \quad I_{l}(x)=i^{-l} J_{l}(i x),
$$

we rewrite the eigenvalue equation (A9) as

$$
\begin{aligned}
& I_{l}\left(\kappa_{\mathrm{II}}\right)\left[\frac{\kappa_{\mathrm{I}}}{\epsilon-\delta_{\mathrm{I}}} K_{l-1}\left(\kappa_{\mathrm{I}}\right)+\frac{\kappa_{\mathrm{I}}}{\epsilon+\delta_{\mathrm{I}}} K_{l+1}\left(\kappa_{\mathrm{I}}\right)\right] \\
& =-K_{l}\left(\kappa_{\mathrm{I}}\right)\left[\frac{\kappa_{\mathrm{II}}}{\epsilon-\delta_{\mathrm{II}}} I_{l-1}\left(\kappa_{\mathrm{II}}\right)+\frac{\kappa_{\mathrm{II}}}{\epsilon+\delta_{\mathrm{II}}} I_{l+1}\left(\kappa_{\mathrm{II}}\right)\right]
\end{aligned}
$$

with the associated eigenstates given by

$$
\begin{aligned}
& \Psi_{l}(\boldsymbol{r})=\left\langle\mathrm{I} \mid \Psi_{l}\right\rangle+\left\langle\mathrm{II} \mid \Psi_{l}\right\rangle \\
& =\frac{\sqrt{2} i^{-l} C_{\mathrm{I}}}{\pi}\left(\begin{array}{c}
\frac{i \kappa_{\mathrm{I}}}{\epsilon-\delta_{\mathrm{I}}} K_{l-1}\left(\kappa_{\mathrm{I}} \rho\right) e^{-i \theta} \\
\sqrt{2} K_{l}\left(\kappa_{\mathrm{I}} \rho\right) \\
\frac{i \kappa_{\mathrm{I}}}{\epsilon+\delta_{\mathrm{I}}} K_{l+1}\left(\kappa_{\mathrm{I}} \rho\right) e^{i \theta}
\end{array}\right) e^{i l \theta} \Theta(r-R) \\
& +\frac{i^{l+1} C_{\mathrm{II}}}{\sqrt{2}}\left(\begin{array}{c}
\frac{-i \kappa_{\mathrm{II}}}{\epsilon-\delta_{\mathrm{II}}} I_{l-1}\left(\kappa_{\mathrm{II}} \rho\right) e^{-i \theta} \\
\sqrt{2} I_{l}\left(\kappa_{\mathrm{II}} \rho\right) \\
\frac{-i \kappa_{\mathrm{II}}}{\epsilon+\delta_{\mathrm{II}}} I_{l+1}\left(\kappa_{\mathrm{II}} \rho\right) e^{i \theta}
\end{array}\right) e^{i l \theta} \Theta(R-r),
\end{aligned}
$$

where $\rho=r / R, I_{l}(x)$ and $K_{l}(x)$ are modified Bessel functions.

As explained in the main text, a surprising phenomenon uncovered through the solutions as described above is the emergence of in-gap chiral edge modes on the confinement boundary without imposing any topological band inversion. This breaks the widely believed principle of bulk-edge correspondence. Especially, this principle stipulates that only domainwall configurations with band inversion, i.e., $\Delta_{\mathrm{I}} \Delta_{\mathrm{II}}<0$, can bear chiral in-gap edge states. Figure 8 shows some representative results. For reference, we also include the corresponding results for the conventional, massive spin- $\frac{1}{2}$ Dirac fermion system. We see that, for the spin- 1 system, a branch of chiral edge modes exists for the noninverted case, but no such nodes can arise in the spin- $\frac{1}{2}$ Dirac fermion system or other existing wave systems [44-46,60].

Existence of chiral zero-energy modes: A rigorous proof. An intriguing phenomenon in Fig. 8 is the emergence of the zero-energy modes. The existence of these modes can be proved rigorously, enabling an analytic understanding of their properties. In particular, for $\epsilon=0$, the eigenvalue equation (A11) reduces to

$$
2 l \frac{I_{l}\left(\left|\delta_{\mathrm{II}}\right|\right) K_{l}\left(\left|\delta_{\mathrm{I}}\right|\right)}{\delta_{\mathrm{I}} \delta_{\mathrm{II}}}\left[\delta_{\mathrm{I}}+e^{-i(l-1) \pi} \delta_{\mathrm{II}}\right]=0,
$$



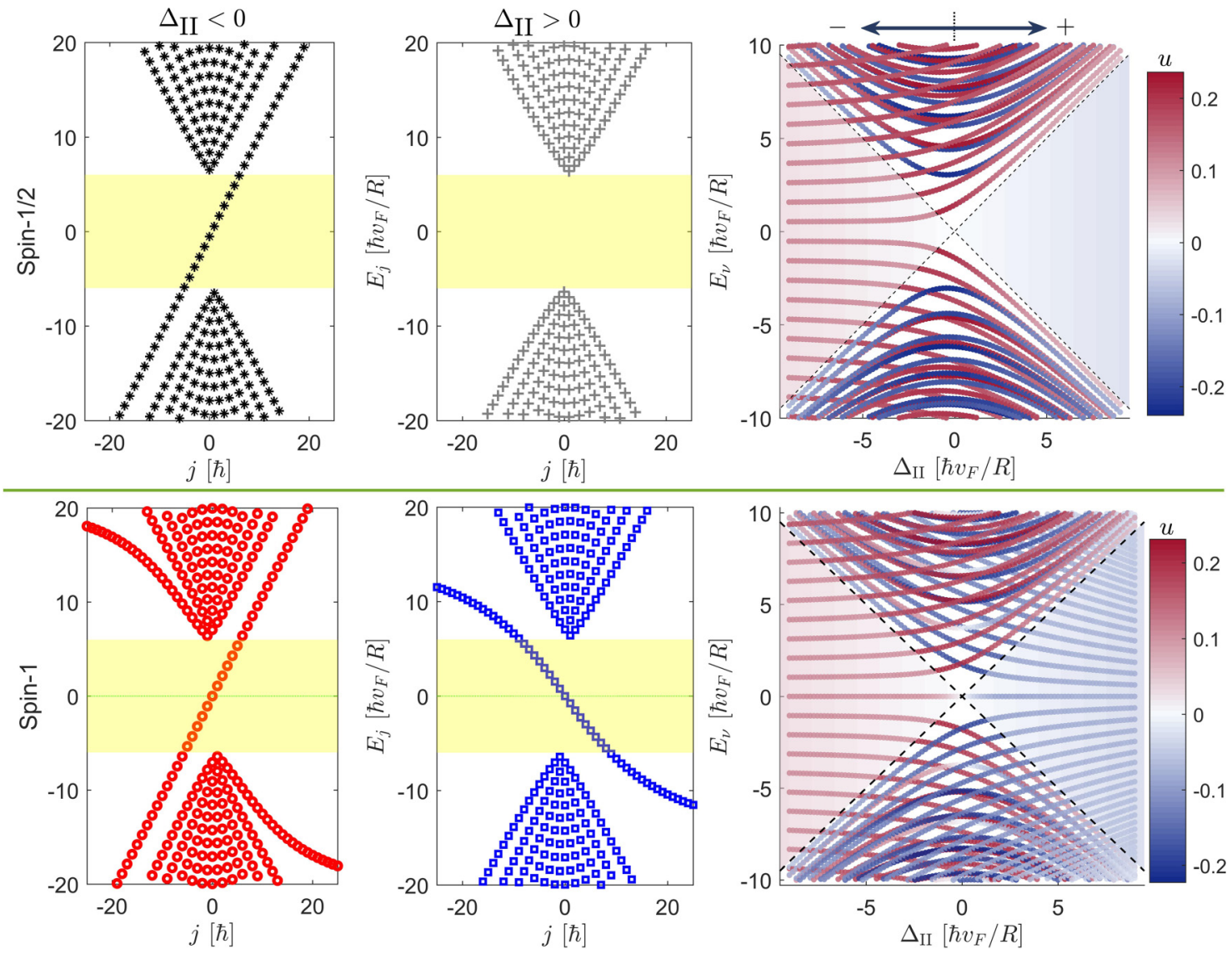

FIG. 8. Eigenspectra of a massive Dirac quantum dot confinement. Top and bottom panels: calculated eigenspectra for a quantum dot of Dirac materials of conventional massive spin- $\frac{1}{2}$ Dirac fermions and spin-1 generalization, respectively. In both cases, the Dirac mass is fixed at $\Delta_{\mathrm{I}}=+36 \hbar v_{F} / R$. The left and middle columns show the eigenenergy versus the angular momentum for the inverted $\left(\Delta_{\mathrm{II}}=-6 \hbar v_{F} / R\right)$ and noninverted $\left(\Delta_{\mathrm{II}}=+6 \hbar v_{F} / R\right)$ confinement cases, respectively. The right column is eigenenergy versus the gap opening (i.e., mass) of the inner domain $\Delta_{\mathrm{II}}$. For spin- $\frac{1}{2}$ Dirac fermions, without band inversion no topological edge states can occur (top middle panel and right half of the top right panel).

which gives an eigensolution for $l=0$, i.e.,

$$
\epsilon=0: \Psi_{0}(\boldsymbol{r})=\Psi_{0}^{\mathrm{I}}(\boldsymbol{r}) \Theta(r-R)+\Psi_{0}^{\mathrm{II}}(\boldsymbol{r}) \Theta(R-r),
$$

with

$$
r>R: \Psi_{0}^{\mathrm{I}}(\boldsymbol{r})=\frac{\sqrt{2} C_{\mathrm{I}}}{\pi}\left(\begin{array}{c}
-i K_{1}\left(\left|\delta_{\mathrm{I}}\right| \rho\right) e^{-i \theta} \\
\operatorname{sgn}\left(\delta_{\mathrm{I}}\right) \sqrt{2} K_{0}\left(\left|\delta_{\mathrm{I}}\right| \rho\right) \\
i K_{1}\left(\left|\delta_{\mathrm{I}}\right| \rho\right) e^{i \theta}
\end{array}\right)
$$

and

$$
r<R: \quad \Psi_{0}^{\mathrm{II}}(\boldsymbol{r})=\frac{i C_{\mathrm{II}}}{\sqrt{2}}\left(\begin{array}{c}
i I_{1}\left(\left|\delta_{\mathrm{II}}\right| \rho\right) e^{-i \theta} \\
\operatorname{sgn}\left(\delta_{\mathrm{II}}\right) \sqrt{2} I_{0}\left(\left|\delta_{\mathrm{II}}\right| \rho\right) \\
-i I_{1}\left(\left|\delta_{\mathrm{II}}\right| \rho\right) e^{i \theta}
\end{array}\right) .
$$

The underlying local current and spin densities are

$$
\begin{gathered}
\boldsymbol{u}^{\mathrm{I}}=\frac{8 v_{F}\left|C_{\mathrm{I}}\right|^{2}}{\pi^{2}} K_{0} K_{1} \times \operatorname{sgn}\left(\delta_{\mathrm{I}}\right)(-\sin \theta, \cos \theta)=\left(u_{x}^{\mathrm{I}}, u_{y}^{\mathrm{I}}\right), \\
\boldsymbol{u}^{\mathrm{II}}=2 v_{F}\left|C_{\mathrm{II}}\right|^{2} I_{0} I_{1} \times \operatorname{sgn}\left(\delta_{\mathrm{II}}\right)(\sin \theta,-\cos \theta)=\left(u_{x}^{\mathrm{II}}, u_{y}^{\mathrm{II}}\right)
\end{gathered}
$$

and

$$
\begin{aligned}
S^{\mathrm{I}} & =\frac{8\left|C_{\mathrm{I}}\right|^{2}}{\pi^{2}} K_{0} K_{1} \times \operatorname{sgn}\left(\delta_{\mathrm{I}}\right)(-\sin \theta, \cos \theta, 0)=\left(S_{x}^{\mathrm{I}}, S_{y}^{\mathrm{I}}, S_{z}^{\mathrm{I}}\right), \\
S^{\mathrm{II}} & =2\left|C_{\mathrm{II}}\right|^{2} I_{0} I_{1} \times \operatorname{sgn}\left(\delta_{\mathrm{II}}\right)(\sin \theta,-\cos \theta, 0)=\left(S_{x}^{\mathrm{II}}, S_{y}^{\mathrm{II}}, S_{z}^{\mathrm{II}}\right) .
\end{aligned}
$$

We see that, for the zero-energy modes, the spin textures are fully in-plane polarized and perfectly locked with the currents, regardless of whether there is topological band inversion (i.e., $\left.\delta_{\mathrm{I}} \delta_{\mathrm{II}}<0\right)$ or not.

Current-carrying properties and dispersion relation between energy and angular momentum. The current-carrying properties of the states can be inferred from the dispersion relation between energy and angular momentum, and vice versa. In particular, with the ansatz in Eq. (A6), the generalized Dirac equation in the radial direction deduced from Eq. (A5) is

$$
\hat{H}_{r} \mathcal{R}_{l}(r)=\epsilon_{l} \mathcal{R}_{l}(r),
$$


where $\mathcal{R}_{l}(r)=\left[\mathcal{R}_{1}, \mathcal{R}_{2}, \mathcal{R}_{3}\right]^{T}$ with the radial Hamiltonian given by

$$
\hat{H}_{r}=\left(\begin{array}{ccc}
\delta & -\frac{i}{\sqrt{2}}\left(\frac{d}{d r}+\frac{l}{r}\right) & 0 \\
-\frac{i}{\sqrt{2}}\left(\frac{d}{d r}-\frac{l-1}{r}\right) & 0 & -\frac{i}{\sqrt{2}}\left(\frac{d}{d r}+\frac{l+1}{r}\right) \\
0 & -\frac{i}{\sqrt{2}}\left(\frac{d}{d r}-\frac{l}{r}\right) & -\delta
\end{array}\right)
$$

Making use of the Hellmann-Feynman theorem [61], we get

$$
\begin{aligned}
\frac{\partial \epsilon_{l}}{\partial l}=\left\langle\mathcal{R}_{l}\left|\frac{\partial \hat{H}_{r}}{\partial l}\right| \mathcal{R}_{l}\right\rangle & =\int_{0}^{\infty} r d r\left(\mathcal{R}_{1}^{*}, \mathcal{R}_{2}^{*}, \mathcal{R}_{3}\right)\left(\begin{array}{ccc}
0 & -\frac{i}{r \sqrt{2}} & 0 \\
\frac{i}{r \sqrt{2}} & 0 & -\frac{i}{r \sqrt{2}} \\
0 & \frac{i}{r \sqrt{2}} & 0
\end{array}\right)\left(\begin{array}{l}
\mathcal{R}_{1} \\
\mathcal{R}_{2} \\
\mathcal{R}_{3}
\end{array}\right) \\
& =\int_{0}^{\infty} d r\left\{-\sqrt{2} \operatorname{Im}\left[\mathcal{R}_{2}^{*}\left(\mathcal{R}_{1}-\mathcal{R}_{3}\right)\right]\right\} \equiv \int_{0}^{\infty} d r \frac{u_{\theta}}{v_{F}}=\frac{u}{v_{F}},
\end{aligned}
$$

which relates, in an explicit way, the current carried by the eigenstates to the derivative of the corresponding eigenenergy with respect to the total angular momentum quantum number $l$.

\section{Landau levels and confinement in the presence of a magnetic field}

We consider an external, perpendicular magnetic field $\boldsymbol{B}=B \hat{\boldsymbol{e}}_{z}$ applied to the 2D quantum dot structure. It is convenient to choose the symmetric gauge: $\boldsymbol{A}=B / 2(-y, x)$. Equation (A5) becomes

$$
\left(\begin{array}{ccc}
\delta & -\frac{i e^{-i \theta}}{\sqrt{2}}\left(\partial_{r}-\frac{i}{r} \partial_{\theta}+b r\right) & 0 \\
-\frac{i e^{i \theta}}{\sqrt{2}}\left(\partial_{r}+\frac{i}{r} \partial_{\theta}-b r\right) & 0 & -\frac{i e^{-i \theta}}{\sqrt{2}}\left(\partial_{r}-\frac{i}{r} \partial_{\theta}+b r\right) \\
0 & -\frac{i e^{i \theta}}{\sqrt{2}}\left(\partial_{r}+\frac{i}{r} \partial_{\theta}-b r\right) & -\delta
\end{array}\right) \Psi_{l}(\boldsymbol{r})=\epsilon \Psi_{l}(\boldsymbol{r}),
$$

where $b=e B / 2 \hbar$ with $e$ being the magnitude of the electronic charge. For a bulk gapped system of a spatially uniform $\delta$ (gap opening), for $\epsilon^{2} \neq \delta^{2}$, we obtain the following decoupled second-order differential equation:

$$
\frac{d^{2} \mathcal{R}_{2}}{d r^{2}}+\frac{1}{r} \frac{d \mathcal{R}_{2}}{d r}-\left[b^{2} r^{2}+a+\frac{n^{2}}{r^{2}}\right] \mathcal{R}_{2}=0,
$$

where

$$
a=2 b(l-\delta / \epsilon)-\left(\epsilon^{2}-\delta^{2}\right)
$$

and $n=|l|$ with $l \in \mathbb{Z}$. The solutions which are regular at the origin are

$$
\mathcal{R}_{2}(r)=c r^{n} e^{-z / 2} M(\gamma, \eta, z),
$$

where $M$ denotes the Kummer $M$ function, $c$ is the coefficient, $z=b r^{2}, \eta=n+1$, and

$$
\gamma=\frac{1}{4}\left[\frac{a}{b}+2(n+1)\right] .
$$

The other two components can be obtained by substituting the expression of $\mathcal{R}_{2}$ back into Eq. (A19). The whole solution set for $l>0$ is given by

$$
\left(\begin{array}{l}
\mathcal{R}_{1} \\
\mathcal{R}_{2} \\
\mathcal{R}_{3}
\end{array}\right)=c_{+} r^{n} e^{-z / 2}\left(\begin{array}{c}
\frac{2 n}{(\epsilon-\delta) r} M(\gamma, n, z) \\
i M(\gamma, n+1, z) \\
-\frac{2 b r(n+1-\gamma)}{(\epsilon+\delta)(n+1)} M(\gamma, n+2, z)
\end{array}\right)
$$

for $l<0$

$$
\left(\begin{array}{l}
\mathcal{R}_{1} \\
\mathcal{R}_{2} \\
\mathcal{R}_{3}
\end{array}\right)=c_{-} r^{n} e^{-z / 2}\left(\begin{array}{c}
\frac{2 b r \gamma}{(\epsilon-\delta)(n+1)} M(\gamma+1, n+2, z) \\
i M(\gamma, n+1, z) \\
-\frac{2 n}{(\epsilon+\delta) r} M(\gamma-1, n, z)
\end{array}\right)
$$


For $l=0$, we have

$$
\left(\begin{array}{l}
\mathcal{R}_{1} \\
\mathcal{R}_{2} \\
\mathcal{R}_{3}
\end{array}\right)=c_{0} e^{-z / 2}\left(\begin{array}{c}
\frac{2 b-\epsilon(\epsilon+\delta)}{2 \epsilon} r M(\gamma+1,2, z) \\
i M(\gamma, 1, z) \\
-\frac{2 b+\epsilon(\epsilon-\delta)}{2 \epsilon} r M(\gamma, 2, z)
\end{array}\right) .
$$

For $\epsilon^{2}-\delta^{2}=0$, Eq. (A19) has another regular solution at $\epsilon=-\delta, l=-1$ with the associated state given by

$$
\left(\begin{array}{l}
\mathcal{R}_{1} \\
\mathcal{R}_{2} \\
\mathcal{R}_{3}
\end{array}\right)=c e^{-z / 2}\left(\begin{array}{l}
0 \\
0 \\
1
\end{array}\right)
$$

Normalizability of $M(\gamma, \eta, z)$ requires $\gamma$ to be a nonpositive integer $-N=0,-1,-2, \ldots$, which leads to Landau levels

$$
E_{\mathrm{LL}}= \begin{cases}-\Delta, & \text { for } l=-1 \\ \frac{\hbar v_{F}}{R} \frac{-\delta \pm \sqrt{\delta^{2}+4\left(R / l_{B}\right)^{2}}}{2}, & \text { for } l=0 \\ 2 \frac{\hbar v_{F}}{R} \sqrt{-\frac{p}{3}} \cos \left[\frac{1}{3} \arccos \left(\frac{3 q}{2 p} \sqrt{\frac{-3}{p}}\right)-v \frac{2 \pi}{3}\right], & \text { for } l \neq 0\end{cases}
$$

where $v=0,1,2$, and

$$
p=-\left[\delta^{2}+\left(\frac{R}{l_{B}}\right)^{2}(2 N+l+|l|+1)\right], \quad q=\delta\left(\frac{R}{l_{B}}\right)^{2}
$$

with the magnetic length defined as $l_{B}=\sqrt{\hbar / e B}$.

In the presence of the confining potential $\Delta(r)$, to solve the system analytically is infeasible due to the complex interplay between confinement mechanisms due to the dot geometry and the Landau levels. We thus develop a numerical solver for massive spin-1 particles based on the finite-difference method (FDM) originally designed for conventional spin- $\frac{1}{2}$ Dirac fermion systems [62-65]. In particular, using the polar decomposition ansatz

$$
\psi_{l}(r, \theta)=\frac{e^{i l \theta}}{\sqrt{r}}\left(\begin{array}{c}
\widetilde{\mathcal{R}}_{1}(r) e^{-i \theta} \\
\widetilde{\mathcal{R}}_{2}(r) \\
\widetilde{\mathcal{R}}_{3}(r) e^{i \theta}
\end{array}\right),
$$

we obtain the corresponding radial eigenvalue equation for the three-component spinor $\widetilde{\mathcal{R}}=\left[\widetilde{\mathcal{R}}_{1}, \widetilde{\mathcal{R}}_{2}, \widetilde{\mathcal{R}}_{3}\right]^{T}$ as

$$
\hat{H}_{r} \widetilde{\mathcal{R}}=\left[-i \hat{S}_{x} \partial_{r}+\hat{S}_{y}\left(\frac{l}{r}+b r\right)-\frac{1 / 2}{r}\left(\begin{array}{ccc}
0 & -i / \sqrt{2} & 0 \\
i / \sqrt{2} & 0 & i / \sqrt{2} \\
0 & -i / \sqrt{2} & 0
\end{array}\right)+\hat{S}_{z} \frac{\Delta(r)}{\hbar v_{F}}\right] \widetilde{\mathcal{R}}=\frac{E}{\hbar v_{F}} \widetilde{\mathcal{R}} .
$$

When discretizing this equation on a finite lattice/grid, it is necessary to judiciously specify the difference scheme and the boundary conditions at the ends of the lattice so as to preserve the Hermiticity of the Hamiltonian. An effective procedure is the backward-forward-backward (BFB) difference scheme to approximate the derivatives of the three components in Eq. (A27):

$$
\partial_{r} \widetilde{\mathcal{R}}_{1} \approx \frac{\widetilde{\mathcal{R}}_{1}(r)-\widetilde{\mathcal{R}}_{1}(r-h)}{h}, \quad \partial_{r} \widetilde{\mathcal{R}}_{2} \approx \frac{\widetilde{\mathcal{R}}_{2}(r+h)-\widetilde{\mathcal{R}}_{2}(r)}{h}, \quad \partial_{r} \widetilde{\mathcal{R}}_{3} \approx \frac{\widetilde{\mathcal{R}}_{3}(r)-\widetilde{\mathcal{R}}_{3}(r-h)}{h},
$$

where $h=L /(N+1)$ is the discretization step size for the system in the range $0<r<L$ with $N+2$ lattice points. The boundary conditions can be deduced from the Hermitian constraint of $\hat{H}_{r}$ :

$$
\int_{0}^{L}\left[\widetilde{\mathcal{R}}_{\alpha}^{\dagger} \hat{H}_{r} \widetilde{\mathcal{R}}_{\beta}-\left(\hat{H}_{r} \widetilde{\mathcal{R}}_{\alpha}\right)^{\dagger} \widetilde{\mathcal{R}}_{\beta}\right] d r=0,
$$

which can be explicitly written as

$$
-\left.\frac{i}{\sqrt{2}}\left[\left(\widetilde{\mathcal{R}}_{1 \alpha}+\widetilde{\mathcal{R}}_{3 \alpha}\right)^{*} \widetilde{\mathcal{R}}_{2 \beta}+\widetilde{\mathcal{R}}_{2 \alpha}^{*}\left(\widetilde{\mathcal{R}}_{1 \beta}+\widetilde{\mathcal{R}}_{3 \beta}\right)\right]\right|_{0} ^{L}=0 .
$$

The specific boundary conditions on $\widetilde{\mathcal{R}}(0)$ and $\widetilde{\mathcal{R}}(L)$ then become

$$
\widetilde{\mathcal{R}}_{1}(0)+\widetilde{\mathcal{R}}_{3}(0)=0, \quad \widetilde{\mathcal{R}}_{2}(L)=0 .
$$

Implementing this procedure results in an eigenvalue problem for a $3 N \times 3 N$ Hermitian matrix $\mathbb{H}_{3 N \times 3 N}=\left[H_{\mu \nu}\right]$ with elements given by

$$
\begin{aligned}
H_{(3 n-2) \times(3 n-2)} & =\delta_{n}, \quad H_{(3 n-1) \times(3 n-1)}=0, \quad H_{3 n \times 3 n}=-\delta_{n}, \\
H_{(3 n-2) \times(3 n-1)} & =\frac{i}{\sqrt{2} h}-\frac{i}{\sqrt{2}}\left(\frac{l-1 / 2}{r_{n}}+b r_{n}\right), \\
H_{(3 n-1) \times(3 n-2)} & =\left(H_{(3 n-2) \times(3 n-1)}\right)^{*}, \\
H_{(3 n-1) \times 3 n} & =-\frac{i}{\sqrt{2} h}-\frac{i}{\sqrt{2}}\left(\frac{l+1 / 2}{r_{n}}+b r_{n}\right), \\
H_{3 n \times(3 n-1)} & =\left(H_{(3 n-1) \times 3 n}\right)^{*},
\end{aligned}
$$


(a)

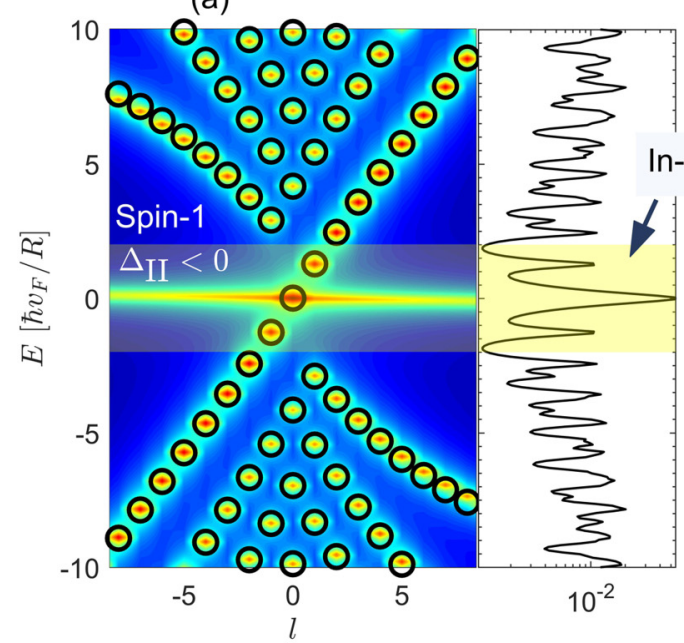

(c)

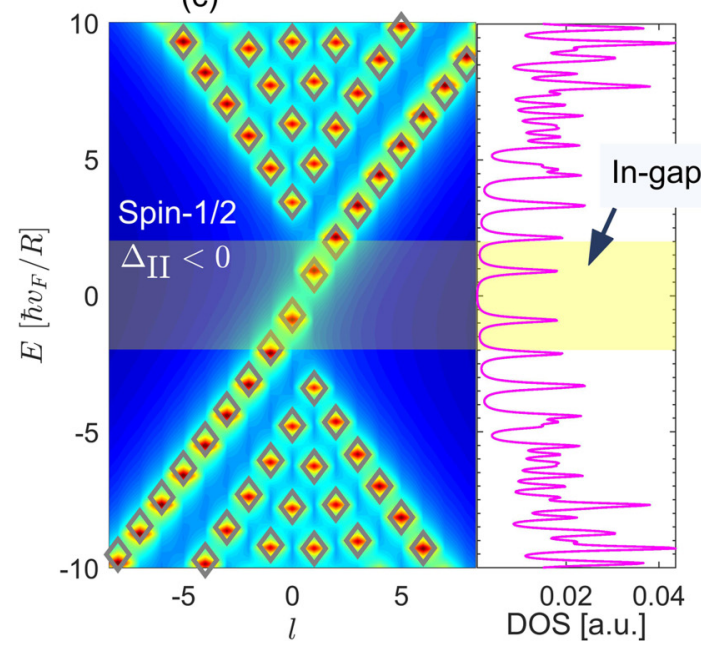

(b)

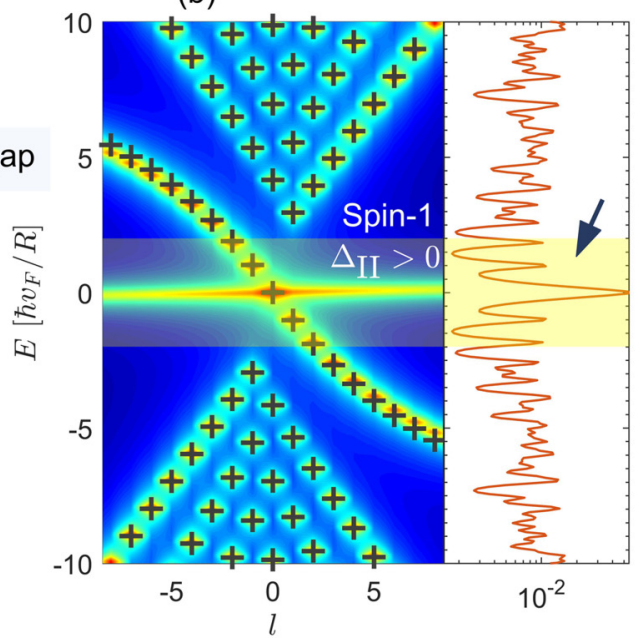

(d)

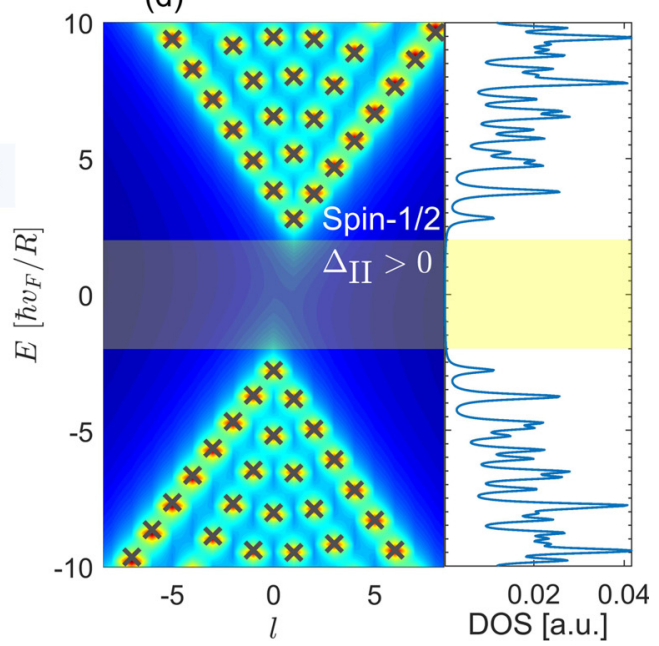

FIG. 9. Validation of finite-difference method for solving the generalized spin-1 Dirac quantum dot confinement systems. Shown are comparisons between numerical and analytic results: contour map of the density of states (DOS) versus energy and angular momentum for $\boldsymbol{A}=0$, where the analytic solutions are represented by the markers. For fixed value of $\Delta_{\mathrm{I}}=+20 \hbar v_{F} / R$, the cases with and without band inversion are specified by $\Delta_{\mathrm{II}}=-2 \hbar v_{F} / R$ and $\Delta_{\mathrm{II}}=+2 \hbar v_{F} / R$, respectively.

for $n=1, \ldots, N$. For $n<N$, the matrix elements are

$$
H_{(3 n-2) \times[3(n+1)-1]}=-\frac{i}{\sqrt{2} h}, \quad H_{[3(n+1)-1] \times(3 n-2)}=\frac{i}{\sqrt{2} h}, \quad H_{3 n \times[3(n+1)-1]}=-\frac{i}{\sqrt{2} h}, \quad H_{[3(n+1)-1] \times 3 n}=\frac{i}{\sqrt{2} h} .
$$

We use the typical experimental values of the local density of states (DOS) $[62,64,65]$ to measure the spectral features, which are defined as

$$
D\left(E, r_{0}\right)=\sum_{l} \sum_{v} \frac{\Gamma}{\pi} \frac{\left\langle\left|\mathcal{R}_{v}\left(r=r_{0}\right)\right|^{2}\right\rangle_{\lambda}}{\left(E-E_{l v}\right)^{2}+\Gamma^{2}}
$$

with $v$ labeling the radial eigenstates for fixed $l$, and

$$
\left\langle\left|\mathcal{R}_{v}\left(r=r_{0}\right)\right|^{2}\right\rangle_{\lambda}=\int_{0}^{L} d r\left|\mathcal{R}_{v}(r)\right|^{2} e^{-\left(r-r_{0}\right)^{2} / 2 \lambda}
$$

representing the spatial average of the wave function centered at $r=r_{0}$ with a Gaussian weight $\lambda$. We approximate the $\delta$ function by a Lorentzian with the broadening parameter $\Gamma$. For illustrative purpose, in our simulations, we use a system of size $L / R=10$ and discretize it with a uniform lattice of $N=600$ sites. Other parameters are chosen as $\Gamma / E_{*}=0.2$ and $\lambda=0.1 R$. Representative results are shown in Figs. 9 and 10, which agree with the analytic results (in special cases where they are available) very well. Physically, the results provide strong support for the predicted emergence of unconventional in-gap chiral edge modes in gapped spin-1 Dirac systems without topological band inversion. 
(a)

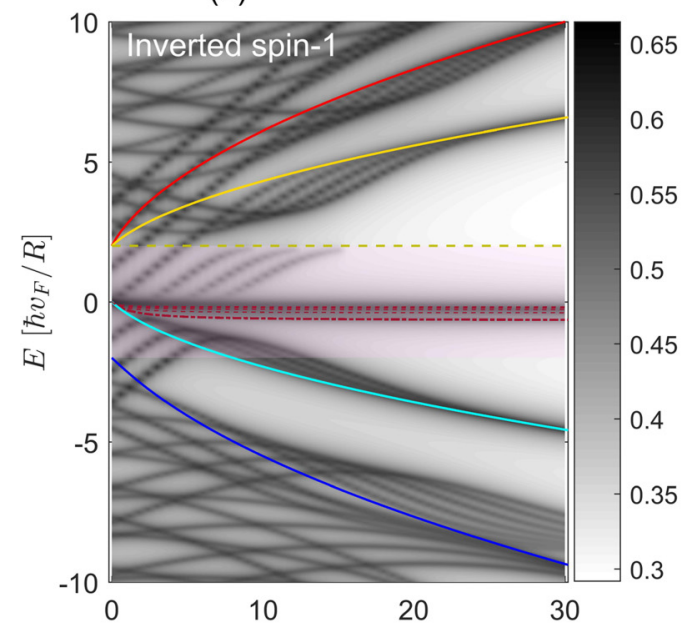

(c)

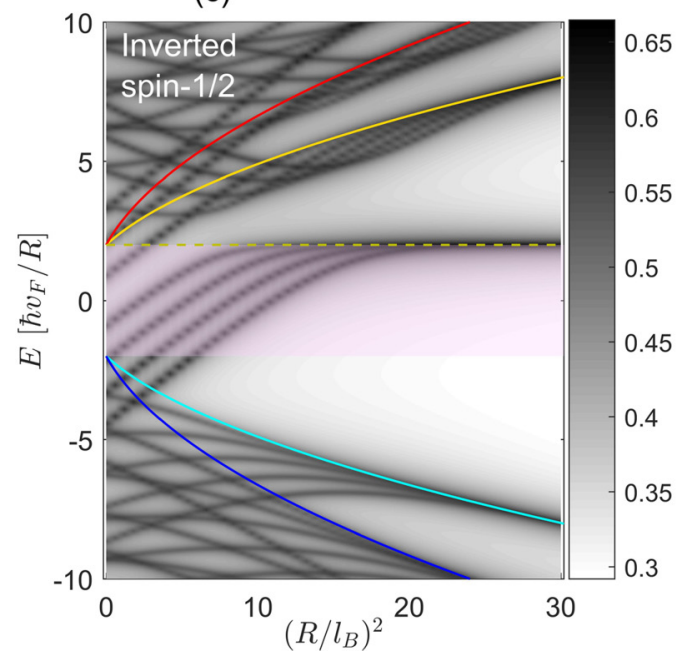

(b)

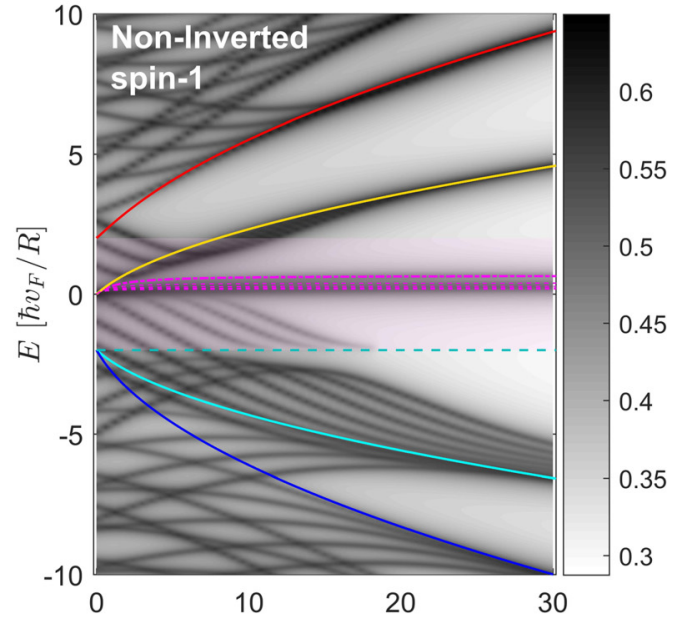

(d)

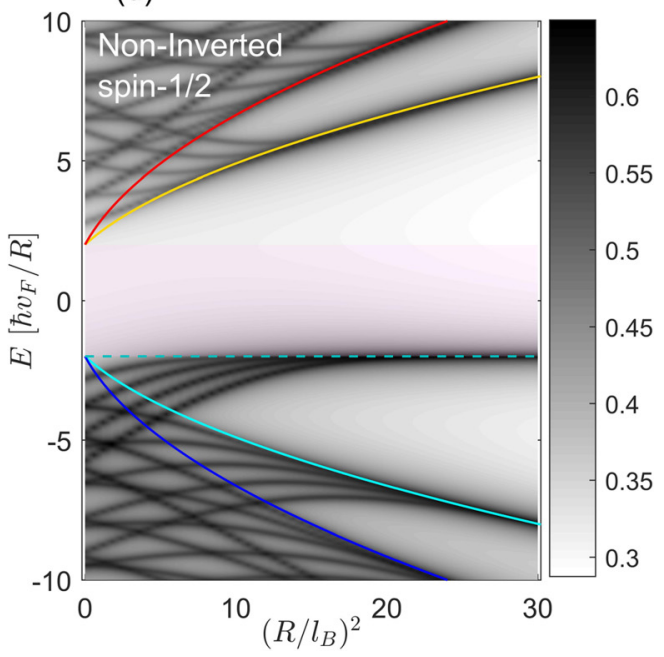

FIG. 10. Evolution of eigenenergy as a function of the applied magnetic field in dot confinement. The field is parametrized by $\left(R / l_{B}\right)^{2}$. The left and right columns are for the cases where there is and is no band inversion: $\Delta_{\mathrm{II}}=-2 \hbar v_{F} / R$ and $\Delta_{\mathrm{II}}=+2 \hbar v_{F} / R$, respectively, with respect to the fixed gap opening of the outer domain $\Delta_{\mathrm{I}}=+20 \hbar v_{F} / R$. For high magnetic fields, the results converge to the Landau level spectrum indicated by colored curves.

\section{APPENDIX B: MULTIPLE MULTIPOLES METHOD: CALCULATION OF EIGENENERGIES AND EIGENSTATES OF MASSIVE SPIN-1 DIRAC PARTICLE IN ARBITRARY DOMAINS}

For an arbitrarily shaped dot geometry, we develop a multiple expansion technique to solve the Dirac-Weyl equation for spin-1 particles, incorporating the evanescent waves by generalizing the multiple multipole expansion method originally developed in optics [66-70]. Our method is computationally reliable and efficient, providing a powerful tool to detect and verify the existence of in-gap excitations/modes and study their robustness in the presence of geometric deformations.

\section{Implementation}

A concrete setting of a single potential domain of arbitrary shape is illustrated in Fig. 11, where the exact shape of the geometric boundary is specified according to the superformula in botany [71], a simple but powerful prescription that can generate a vast variety of complex geometric shapes. In the polar coordinates, the superformula is

$$
r(\theta)=\left[\left|\frac{1}{a} \cos \left(\frac{m_{1}}{4} \theta\right)\right|^{n_{2}}+\left|\frac{1}{b} \cos \left(\frac{m_{2}}{4} \theta\right)\right|^{n_{3}}\right]^{-1 / n_{1}},
$$

where the parameters $\left(m_{1}, m_{2}, n_{1}, n_{2}, n_{3} ; a, b\right)$ control the shape. The boundary defines two subregions, one exterior another interior, denoted by I and II, respectively, as shown in Fig. 11. The three-component spinor wave equation for a massive spin-1 Dirac particle in each sub-region $\tau \in\{\mathrm{I}, \mathrm{II}\}$ reads as

$$
\left[\hat{\boldsymbol{S}} \cdot \hat{\boldsymbol{k}}+\delta_{\tau} S_{z}\right] \Psi^{(\tau)}(\boldsymbol{r})=\epsilon \Psi^{(\tau)}(\boldsymbol{r}),
$$

where $\delta_{\tau}=\Delta_{\tau} / \hbar v_{F}$ and $\epsilon=E / \hbar v_{F}$. In the polar coordinates $\boldsymbol{r}=(r, \theta)$, the spinor cylindrical wave basis of the solutions 


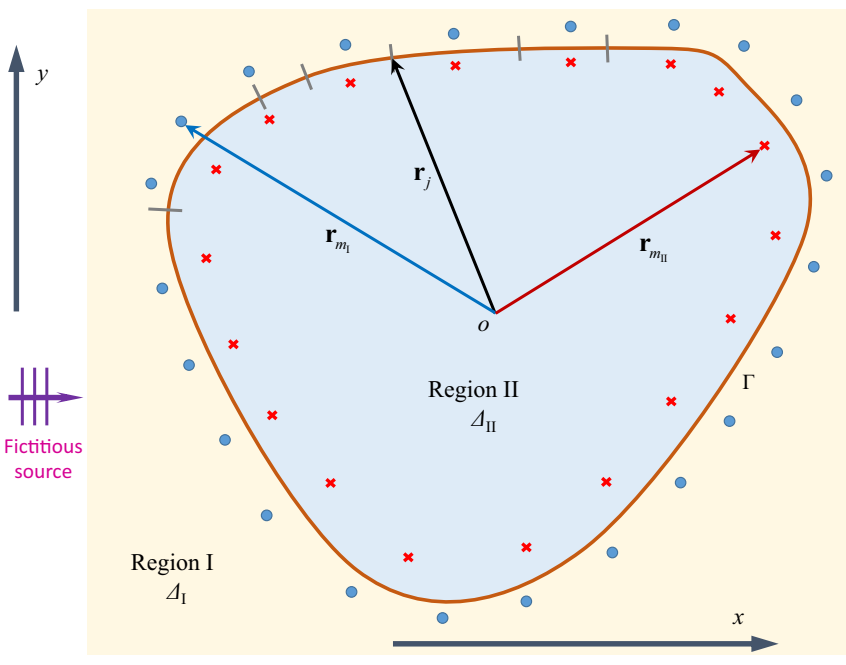

FIG. 11. Schematic illustration of the setting of multiple multipole expansion method. The confining potential has a closed boundary $\Gamma$ separating regions I and II. The basis functions originated at $\boldsymbol{r}_{m_{\mathrm{I}}}$ (blue circular dots) are used to determine the wave function in region II, while those at $\boldsymbol{r}_{m_{\mathrm{II}}}$ (red crosses) determine the wave function in region I. The boundary conditions for the massive spin-1 Dirac wave functions are imposed at the collocation points $\boldsymbol{r}_{j} \in \Gamma$.

with angular momentum $l$ is

$$
\Psi_{l}^{(\tau)}(\boldsymbol{r})=\frac{1}{\sqrt{2}}\left(\begin{array}{c}
\alpha_{\tau} B_{l-1}\left(k_{\tau} r\right) e^{-i \theta} \\
i \sqrt{2} B_{l}\left(k_{\tau} r\right) \\
-\beta_{\tau} B_{l+1}\left(k_{\tau} r\right) e^{i \theta}
\end{array}\right) e^{i l \theta},
$$

where $\quad \alpha_{\tau}=k_{\tau} /\left(\epsilon-\delta_{\tau}\right), \quad \beta_{\tau}=k_{\tau} /\left(\epsilon+\delta_{\tau}\right), \quad$ and $\quad k_{\tau}=$ $\sqrt{\epsilon^{2}-\delta_{\tau}^{2}}$. Choosing $B_{l}\left(k_{\tau} r\right)=H_{l}^{(1)}\left(k_{\tau} r\right)$ (with $H_{l}^{(1)}$ being the Hankel function of the first kind), we have that the Dirac-type expansion basis wave functions originated at $\boldsymbol{r}_{m_{\bar{\tau}}}$ for the specific region $\tau$ are given by

$$
\Psi_{l}^{(\tau)}\left(\boldsymbol{d}_{m_{\bar{\tau}}}\right)=\frac{1}{\sqrt{2}}\left(\begin{array}{c}
\alpha_{\tau} H_{l-1}^{(1)}\left(k_{\tau} d_{m_{\bar{\tau}}}\right) e^{-i \theta_{m_{\bar{\tau}}}} \\
i \sqrt{2} H_{l}^{(1)}\left(k_{\tau} d_{m_{\bar{\tau}}}\right) \\
-\beta_{\tau} H_{l+1}^{(1)}\left(k_{\tau} d_{m_{\bar{\tau}}}\right) e^{i \theta_{m_{\bar{\tau}}}}
\end{array}\right) e^{i l \theta_{m_{\bar{\tau}}}}
$$

where $\bar{\tau}$ denotes the complement of $\tau$,

$$
d_{m_{\bar{\tau}}} \equiv\left|\boldsymbol{d}_{m_{\bar{\tau}}}\right|=\left|\boldsymbol{r}-\boldsymbol{r}_{m_{\bar{\tau}}}\right|
$$

and

$$
\theta_{m_{\bar{\tau}}}=\operatorname{Angle}\left(\boldsymbol{r}-\boldsymbol{r}_{m_{\bar{\tau}}}\right)
$$

with $\boldsymbol{r} \in \tau$. Carrying out the expansion in region II, we obtain the wave function as

$$
\begin{aligned}
\Psi^{(\mathrm{II})}(\boldsymbol{r}) & =\sum_{m_{\mathrm{I}}} \sum_{l} C_{l}^{m_{\mathrm{I}}} \frac{1}{\sqrt{2}}\left(\begin{array}{c}
\alpha_{\mathrm{II}} H_{l-1}^{(1)}\left(k_{\mathrm{II}} d_{m_{\mathrm{I}}}\right) e^{-i \theta_{m_{\mathrm{I}}}} \\
i \sqrt{2} H_{l}^{(1)}\left(k_{\mathrm{II}} d_{m_{\mathrm{I}}}\right) \\
-\beta_{\mathrm{II}} H_{l+1}^{(1)}\left(k_{\mathrm{II}} d_{m_{\mathrm{I}}}\right) e^{i \theta_{m_{\mathrm{I}}}}
\end{array}\right) e^{i l \theta_{m_{\mathrm{I}}}} \\
& \equiv\left(\begin{array}{c}
\psi_{1}^{\mathrm{II}} \\
\psi_{2}^{\mathrm{II}} \\
\psi_{3}^{\mathrm{II}}
\end{array}\right) .
\end{aligned}
$$

The wave function in region I has the form

$$
\begin{aligned}
& \Psi^{(\mathrm{I})}(\boldsymbol{r})=\sum_{m_{\mathrm{II}}} \sum_{l} C_{l}^{m_{\mathrm{II}}} \frac{1}{\sqrt{2}}\left(\begin{array}{c}
\alpha_{\mathrm{I}} H_{l-1}^{(1)}\left(k_{\mathrm{I}} d_{m_{\mathrm{II}}}\right) e^{-i \theta_{m_{\mathrm{II}}}} \\
i \sqrt{2} H_{l}^{(1)}\left(k_{\mathrm{I}} d_{m_{\mathrm{II}}}\right) \\
-\beta_{\mathrm{I}} H_{l+1}^{(1)}\left(k_{\mathrm{I}} d_{m_{\mathrm{II}}}\right) e^{i \theta_{m_{\mathrm{II}}}}
\end{array}\right) e^{i l \theta_{m_{\mathrm{II}}}} \\
& +\Psi^{\mathrm{in}}(\boldsymbol{r}) \equiv\left(\begin{array}{c}
\psi_{1}^{\mathrm{I}} \\
\psi_{2}^{\mathrm{I}} \\
\psi_{3}^{\mathrm{I}}
\end{array}\right) \text {, }
\end{aligned}
$$

where

$$
\Psi^{\text {in }}(\boldsymbol{r})=\frac{1}{2}\left(\begin{array}{c}
\alpha_{\mathrm{I}} \\
\sqrt{2} \\
\beta_{\mathrm{I}}
\end{array}\right) e^{i k_{\mathrm{I}}\left(x-x_{0}\right)}=\left(\begin{array}{c}
\psi_{1}^{\text {in }} \\
\psi_{2}^{\text {in }} \\
\psi_{3}^{\text {in }}
\end{array}\right)
$$

denotes the input source triggered by an applied external excitation outside of the domain (cf. top panel of Fig. 11).

Imposing the relevant boundary conditions parametrized by the angle $\phi$ between the outward normal at any boundary point $\boldsymbol{r}_{j}$ and the $x$ axis,

$$
\begin{gathered}
\left.\psi_{2}^{(\mathrm{I})}\right|_{\boldsymbol{r}_{j} \in \Gamma}=\left.\psi_{2}^{(\mathrm{II})}\right|_{\boldsymbol{r}_{j} \in \Gamma}, \\
\left.\left(\psi_{1}^{(\mathrm{I})} e^{i \phi}+\psi_{3}^{(\mathrm{I})} e^{-i \phi}\right)\right|_{\boldsymbol{r}_{j} \in \Gamma}=\left.\left(\psi_{1}^{(\mathrm{II})} e^{i \phi}+\psi_{3}^{(\mathrm{II})} e^{-i \phi}\right)\right|_{\boldsymbol{r}_{j} \in \Gamma},
\end{gathered}
$$

we obtain

$$
\begin{aligned}
& \sum_{m_{\mathrm{II}}} \sum_{l}{ }^{j} A_{l m_{\mathrm{II}}}^{(\mathrm{I})} C_{l}^{m_{\mathrm{II}}}-\sum_{m_{\mathrm{I}}} \sum_{l}{ }^{j} A_{l m_{\mathrm{I}}}^{(\mathrm{II})} C_{l}^{m_{\mathrm{I}}}=-{ }^{j} \psi_{2}^{\mathrm{in}}, \\
& \sum_{m_{\mathrm{II}}} \sum_{l}{ }^{j} B_{l m_{\mathrm{II}}}^{(\mathrm{I})} C_{l}^{m_{\mathrm{II}}}-\sum_{m_{\mathrm{I}}} \sum_{l}{ }^{j} B_{l m_{\mathrm{I}}}^{(\mathrm{II})} C_{l}^{m_{\mathrm{I}}}=-{ }^{j} \chi^{\mathrm{in},}
\end{aligned}
$$

where the substitutions are given by

$$
\begin{aligned}
& { }^{j} A_{l m_{\mathrm{II}}}^{(\mathrm{I})}=i H_{l}^{(1)}\left(k_{I}\left|\boldsymbol{r}_{j}-\boldsymbol{r}_{m_{\mathrm{II}}}\right|\right) e^{i l \theta_{m_{\mathrm{II}}}}, \\
& { }^{j} A_{l m_{\mathrm{I}}}^{(\mathrm{II})}=i H_{l}^{(1)}\left(k_{\mathrm{II}}\left|\boldsymbol{r}_{j}-\boldsymbol{r}_{m_{\mathrm{I}}}\right|\right) e^{i l \theta_{m_{\mathrm{I}}}} \\
& { }^{j} B_{l m_{\mathrm{II}}}^{(\mathrm{I})}=\frac{1}{\sqrt{2}}\left[\alpha_{\mathrm{I}} H_{l-1}^{(1)}\left(k_{\mathrm{I}}\left|\boldsymbol{r}_{j}-\boldsymbol{r}_{m_{\mathrm{II}}}\right|\right) e^{i(l-1) \theta_{m_{\mathrm{II}}}} e^{i \phi}-\beta_{\mathrm{I}} H_{l+1}^{(1)}\left(k_{\mathrm{I}}\left|\boldsymbol{r}_{j}-\boldsymbol{r}_{m_{\mathrm{I}}}\right|\right) e^{i(l+1) \theta_{m_{\mathrm{I}}}} e^{-i \phi}\right], \\
& { }^{j} B_{l m_{\mathrm{I}}}^{(\mathrm{II})}=\frac{1}{\sqrt{2}}\left[\alpha_{\mathrm{II}} H_{l-1}^{(1)}\left(k_{\mathrm{II}}\left|\boldsymbol{r}_{j}-\boldsymbol{r}_{m_{\mathrm{I}}}\right|\right) e^{i(l-1) \theta_{m_{\mathrm{I}}}} e^{i \phi}-\beta_{\mathrm{II}} H_{l+1}^{(1)}\left(k_{\mathrm{II}}\left|\boldsymbol{r}_{j}-\boldsymbol{r}_{m_{\mathrm{I}}}\right|\right) e^{i(l+1) \theta_{m_{\mathrm{I}}}} e^{-i \phi}\right],
\end{aligned}
$$


and

$$
\begin{aligned}
{ }^{j} \psi_{2}^{\text {in }} & =\frac{1}{\sqrt{2}} e^{i k_{\mathrm{I}}\left(\left|\boldsymbol{r}_{j}\right| \cos \theta_{j}-x_{0}\right)}, \\
{ }^{j} \chi^{\text {in }} & =\frac{1}{2}\left[\alpha_{\mathrm{I}} e^{i \phi}+\beta_{\mathrm{I}} e^{-i \phi}\right] e^{i k_{\mathrm{I}}\left(\left|\boldsymbol{r}_{j}\right| \cos \theta_{j}-x_{0}\right)} .
\end{aligned}
$$

For the boundary shape defined by Eq. (B1), the associated unit normal direction can be written explicitly

$$
e^{i \phi}=-i e^{i \theta} \frac{d r(\theta) / d \theta+i r(\theta)}{|d r(\theta) / d \theta+i r(\theta)|} .
$$

In principle, the set consists of an infinite number of equations with an infinite number of undetermined expansion coefficients $C_{l}^{m_{I I}}$ and $C_{l}^{m_{I}}$. To solve the system numerically, a finite truncation is necessary, which turns out to be feasible in practice by discretizing the boundary to a finite number $J$ of points and setting the number $M_{\tau}$ of basis functions in the specific region $\tau$ and $l \in[-L, L]$ for all the functions. Carrying out the discretization procedure, we arrive at the following finite-dimensional matrix equation:

$$
\mathbb{M}_{2 J \times N} \cdot \boldsymbol{C}_{N \times 1}=-\boldsymbol{Y}_{2 J \times 1},
$$

where $N=(2 L+1) \times\left(M_{\mathrm{I}}+M_{\mathrm{II}}\right)=N_{\mathrm{I}}+N_{\mathrm{II}}$ and the compact substitutions are

$$
\boldsymbol{C}_{N \times 1}=\left(\begin{array}{c}
C_{-L}^{1_{\mathrm{II}}} \\
\vdots \\
C_{l}^{1_{\mathrm{II}}} \\
C_{l}^{2_{\mathrm{II}}} \\
\vdots \\
C_{l}^{M_{\mathrm{II}}} \\
\vdots \\
C_{L}^{M_{\mathrm{II}}} \\
C_{-L}^{1_{\mathrm{I}}} \\
\vdots \\
C_{l}^{1_{\mathrm{I}}} \\
C_{l}^{2_{\mathrm{I}}} \\
\vdots \\
C_{l}^{M_{\mathrm{I}}} \\
\vdots \\
C_{L}^{M_{\mathrm{I}}}
\end{array}{ }_{N \times 1} \quad \boldsymbol{Y}_{2 J \times 1}=\left(\begin{array}{c}
{ }^{1} \psi_{2}^{\mathrm{in}} \\
\vdots \\
{ }^{j} \psi_{2}^{\mathrm{in}} \\
\vdots \\
{ }^{1} \chi_{2}^{\mathrm{in}} \\
\vdots \\
{ }^{\mathrm{in}} \chi^{\mathrm{in}} \\
\vdots \\
{ }^{J} \chi^{\mathrm{in}}
\end{array}{ }_{2 J \times 1}\right.\right.
$$

and

$$
\mathbb{M}_{2 J \times N}=\left(\begin{array}{l|l}
\mathbb{A}^{(\mathrm{I})} & -\mathbb{A}^{(\mathrm{II})} \\
\hline \mathbb{B}^{(\mathrm{I})} & -\mathbb{B}^{(\mathrm{II})}
\end{array}\right)_{2 J \times N},
$$

with

$$
\begin{aligned}
& \mathbb{A}^{(\tau)}=\left(\begin{array}{llllllll}
\boldsymbol{A}_{-L 1_{\bar{\tau}}}^{(\tau)} & \ldots & \boldsymbol{A}_{l 1_{\bar{\tau}}}^{(\tau)} & \boldsymbol{A}_{l 2_{\bar{\tau}}}^{(\tau)} & \ldots & \boldsymbol{A}_{l M_{\bar{\tau}}}^{(\tau)} & \ldots & \boldsymbol{A}_{L M_{\bar{\tau}}}^{(\tau)}
\end{array}\right)_{J \times N_{\bar{\tau}}}, \\
& \mathbb{B}^{(\tau)}=\left(\begin{array}{llllllll}
\boldsymbol{B}_{-L 1_{\bar{\tau}}}^{(\tau)} & \ldots & \boldsymbol{B}_{l 1_{\bar{\tau}}}^{(\tau)} & \boldsymbol{B}_{l 2_{\bar{\tau}}}^{(\tau)} & \ldots & \boldsymbol{B}_{l M_{\bar{\tau}}}^{(\tau)} & \ldots & \boldsymbol{B}_{L M_{\bar{\tau}}}^{(\tau)}
\end{array}\right)_{J \times N_{\bar{\tau}}},
\end{aligned}
$$



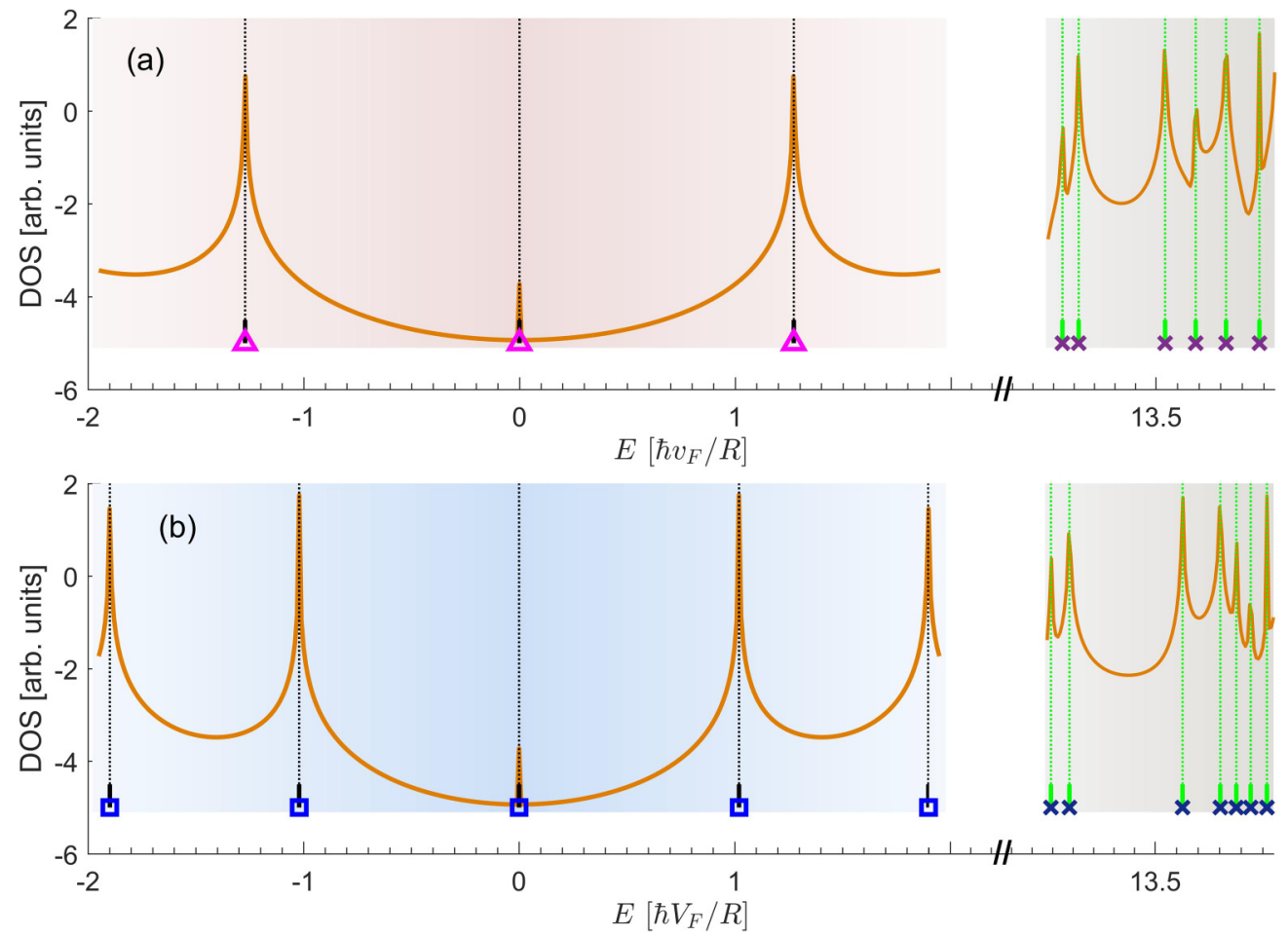

FIG. 12. Validation of the multiple multipole method. For validation purpose, an analytically solvable case of a circular potential domain is used. Comparisons between the numerical results (orange solid lines) calculated from the multiple multipole base expansion method and analytic results (indicated by markers) for (a) case of band inversion: $\Delta_{\mathrm{II}}=-2 \hbar v_{F} / R$ and (b) noninverted case: $\Delta_{\mathrm{II}}=+2 \hbar v_{F} / R$. The reference gap parameter in the exterior domain is $\Delta_{\mathrm{I}}=+20 \hbar v_{F} / R$.

where

$$
\begin{aligned}
\boldsymbol{B}_{l m_{\bar{\tau}}}^{(\tau)} & =\left[{ }^{1} B_{l m_{\bar{\tau}}}^{(\tau)},{ }^{2} B_{l m_{\bar{\tau}}}^{(\tau)}, \ldots,{ }^{j} B_{l m_{\bar{\tau}}}^{(\tau)}, \ldots,{ }^{J} B_{l m_{\bar{\tau}}}^{(\tau)}\right]^{T}, \\
\boldsymbol{A}_{l m_{\bar{\tau}}}^{(\tau)} & =\left[{ }^{1} A_{l m_{\bar{\tau}}}^{(\tau)},{ }^{2} A_{l m_{\bar{\tau}}}^{(\tau)}, \ldots,{ }^{j} A_{l m_{\bar{\tau}}}^{(\tau)}, \ldots,{ }^{J} A_{l m_{\bar{\tau}}}^{(\tau)}\right]^{T} .
\end{aligned}
$$

As the expansions are generally nonorthogonal, more equations are required than the number of unknowns to enable the deduction of an overdetermined matrix system with $2 J \gg N$, which can be solved by the standard pseudoinverse algorithm: $\boldsymbol{C}=-\operatorname{pinv}(\mathbb{M}) * \boldsymbol{Y}$. Particularly, we use the residual error evaluated at the boundary

$$
\text { Error }=\frac{\|\mathbb{M} * \boldsymbol{C}+\boldsymbol{Y}\|}{\|\boldsymbol{Y}\|}
$$

as the criterion to test convergence. We adjust the number, the order, and/or positions of the multipoles to ensure Error $<$ tolerance. After the unknown coefficients $\boldsymbol{C}$ are obtained, the associated wave functions and hence the local density of states in the specific region can be calculated accordingly.

\section{Method validation}

To validate the method, we exploit the analytically solvable case of circular geometry. Figure 12 shows a comparison of the eigenenergy spectra obtained analytically and calculated from the multiple multipole method. The agreement is remarkable.

[1] D. J. Thouless, M. Kohmoto, M. P. Nightingale, and M. den Nijs, Quantized Hall Conductance in A Two-Dimensional Periodic Potential, Phys. Rev. Lett. 49, 405 (1982).

[2] Y. Hatsugai, Chern Number and Edge States in the Integer Quantum Hall Effect, Phys. Rev. Lett. 71, 3697 (1993).

[3] N. R. Cooper, J. Dalibard, and I. B. Spielman, Topological bands for ultracold atoms, Rev. Mod. Phys. 91, 015005 (2019).
[4] T. Ozawa, H. M. Price, A. Amo, N. Goldman, M. Hafezi, L. Lu, M. C. Rechtsman, D. Schuster, J. Simon, O. Zilberberg, and I. Carusotto, Topological photonics, Rev. Mod. Phys. 91, 015006 (2019).

[5] P. Delplace, J. B. Marston, and A. Venaille, Topological origin of equatorial waves, Science 358, 1075 (2017). 
[6] R. Jackiw, Fractional charge and zero modes for planar systems in a magnetic field, Phys. Rev. D 29, 2375 (1984).

[7] R. Jackiw, Fractional and Majorana fermions: The physics of zero-energy modes, Phys. Scr. T146, 014005 (2012).

[8] B. Hunt, J. D. Sanchez-Yamagishi, A. F. Young, M. Yankowitz, B. J. LeRoy, K. Watanabe, T. Taniguchi, P. Moon, M. Koshino, P. Jarillo-Herrero, and R. C. Ashoori, Massive Dirac fermions and Hofstadter butterfly in a van der Waals heterostructure, Science 340, 1427 (2013).

[9] T. Wehling, A. Black-Schaffer, and A. Balatsky, Dirac materials, Adv. Phys. 63, 1 (2014).

[10] Y. Tokura, K. Yasuda, and A. Tsukazaki, Magnetic topological insulators, Nat. Rev. Phys. 1, 126 (2019).

[11] B. Sutherland, Localization of electronic wave functions due to local topology, Phys. Rev. B 34, 5208 (1986).

[12] D. Bercioux, D. F. Urban, H. Grabert, and W. Häusler, Massless Dirac-Weyl fermions in a $T_{3}$ optical lattice, Phys. Rev. A 80, 063603 (2009).

[13] R. Shen, L. B. Shao, B. Wang, and D. Y. Xing, Single Dirac cone with a flat band touching on line-centered-square optical lattices, Phys. Rev. B 81, 041410(R) (2010).

[14] D. Green, L. Santos, and C. Chamon, Isolated flat bands and spin-1 conical bands in two-dimensional lattices, Phys. Rev. B 82, 075104 (2010).

[15] B. Dóra, J. Kailasvuori, and R. Moessner, Lattice generalization of the Dirac equation to general spin and the role of the flat band, Phys. Rev. B 84, 195422 (2011).

[16] F. Wang and Y. Ran, Nearly flat band with Chern number $c=2$ on the dice lattice, Phys. Rev. B 84, 241103(R) (2011).

[17] X. Huang, Y. Lai, Z. H. Hang, H. Zheng, and C. T. Chan, Dirac cones induced by accidental degeneracy in photonic crystals and zero-refractive-index materials, Nat. Mater. 10, 582 (2011).

[18] J. Mei, Y. Wu, C. T. Chan, and Z.-Q. Zhang, First-principles study of Dirac and Dirac-like cones in phononic and photonic crystals, Phys. Rev. B 86, 035141 (2012).

[19] P. Moitra, Y. Yang, Z. Anderson, I. I. Kravchenko, D. P. Briggs, and J. Valentine, Realization of an all-dielectric zero-index optical metamaterial, Nat. Photon. 7, 791 (2013).

[20] A. Raoux, M. Morigi, J.-N. Fuchs, F. Piéchon, and G. Montambaux, From Dia- to Paramagnetic Orbital Susceptibility of Massless Fermions, Phys. Rev. Lett. 112, 026402 (2014).

[21] D. Guzmán-Silva, C. Mejía-Cortés, M. A. Bandres, M. C. Rechtsman, S. Weimann, S. Nolte, M. Segev, A. Szameit, and R. A. Vicencio, Experimental observation of bulk and edge transport in photonic Lieb lattices, New J. Phys. 16, 063061 (2014).

[22] J. Romhányi, K. Penc, and R. Ganesh, Hall effect of triplons in a dimerized quantum magnet, Nat. Commun. 6, 6805 (2015).

[23] G. Giovannetti, M. Capone, J. van den Brink, and C. Ortix, Kekulé textures, pseudospin-one Dirac cones, and quadratic band crossings in a graphene-hexagonal indium chalcogenide bilayer, Phys. Rev. B 91, 121417(R) (2015).

[24] Y. Li, S. Kita, P. Muoz, O. Reshef, D. I. Vulis, M. Yin, M. Lonar, and E. Mazur, On-chip zero-index metamaterials, Nat. Photon. 9, 738 (2015).

[25] S. Mukherjee, A. Spracklen, D. Choudhury, N. Goldman, P. Öhberg, E. Andersson, and R. R. Thomson, Observation of a Localized Flat-Band State in a Photonic Lieb Lattice, Phys. Rev. Lett. 114, 245504 (2015).
[26] R. A. Vicencio, C. Cantillano, L. Morales-Inostroza, B. Real, C. Mejía-Cortés, S. Weimann, A. Szameit, and M. I. Molina, Observation of Localized States in Lieb Photonic Lattices, Phys. Rev. Lett. 114, 245503 (2015).

[27] S. Taie, H. Ozawa, T. Ichinose, T. Nishio, S. Nakajima, and Y. Takahashi, Coherent driving and freezing of bosonic matter wave in an optical Lieb lattice, Sci. Adv. 1, e1500854 (2015).

[28] A. Fang, Z. Q. Zhang, S. G. Louie, and C. T. Chan, Klein tunneling and supercollimation of pseudospin-1 electromagnetic waves, Phys. Rev. B 93, 035422 (2016).

[29] F. Diebel, D. Leykam, S. Kroesen, C. Denz, and A. S. Desyatnikov, Conical Diffraction and Composite Lieb Bosons in Photonic Lattices, Phys. Rev. Lett. 116, 183902 (2016).

[30] L. Zhu, S.-S. Wang, S. Guan, Y. Liu, T. Zhang, G. Chen, and S. A. Yang, Blue phosphorene oxide: Strain-tunable quantum phase transitions and novel 2D emergent fermions, Nano Lett. 16, 6548 (2016).

[31] B. Bradlyn, J. Cano, Z. Wang, M. G. Vergniory, C. Felser, R. J. Cava, and B. A. Bernevig, Beyond Dirac and Weyl fermions: Unconventional quasiparticles in conventional crystals, Science 353, aaf5037 (2016).

[32] I. C. Fulga and A. Stern, Triple point fermions in a minimal symmorphic model, Phys. Rev. B 95, 241116(R) (2017).

[33] M. Ezawa, Triplet fermions and Dirac fermions in borophene, Phys. Rev. B 96, 035425 (2017).

[34] C. Zhong, Y. Chen, Z.-M. Yu, Y. Xie, H. Wang, S. A. Yang, and S. Zhang, Three-dimensional pentagon carbon with a genesis of emergent fermions, Nat. Commun. 8, 15641 (2017).

[35] Y.-Q. Zhu, D.-W. Zhang, H. Yan, D.-Y. Xing, and S.-L. Zhu, Emergent pseudospin-1 Maxwell fermions with a threefold degeneracy in optical lattices, Phys. Rev. A 96, 033634 (2017).

[36] R. Drost, T. Ojanen, A. Harju, and P. Liljeroth, Topological states in engineered atomic lattices, Nat. Phys. 13, 668 (2017).

[37] M. R. Slot, T. S. Gardenier, P. H. Jacobse, G. C. P. van Miert, S. N. Kempkes, S. J. M. Zevenhuizen, C. M. Smith, D. Vanmaekelbergh, and I. Swart, Experimental realization and characterization of an electronic Lieb lattice, Nat. Phys. 13, 672 (2017).

[38] X. Tan, D.-W. Zhang, Q. Liu, G. Xue, H.-F. Yu, Y.-Q. Zhu, H. Yan, S.-L. Zhu, and Y. Yu, Topological Maxwell Metal Bands in a Superconducting Qutrit, Phys. Rev. Lett. 120, 130503 (2018).

[39] Y. Cao, V. Fatemi, S. Fang, K. Watanabe, T. Taniguchi, E. Kaxiras, and P. Jarillo-Herrero, Unconventional superconductivity in magic-angle graphene superlattices, Nature (London) 556, 43 (2018).

[40] M. Yankowitz, S. Chen, H. Polshyn, Y. Zhang, K. Watanabe, T. Taniguchi, D. Graf, A. F. Young, and C. R. Dean, Tuning superconductivity in twisted bilayer graphene, Science 363, 1059 (2019).

[41] A. L. Sharpe, E. J. Fox, A. W. Barnard, J. Finney, K. Watanabe, T. Taniguchi, M. A. Kastner, and D. Goldhaber-Gordon, Emergent ferromagnetism near three-quarters filling in twisted bilayer graphene, Science 365, 605 (2019).

[42] X. Lu, P. Stepanov, W. Yang, M. Xie, M. A. Aamir, I. Das, C. Urgell, K. Watanabe, T. Taniguchi, G. Zhang, A. Bachtold, A. H. MacDonald, and D. K. Efetov, Superconductors, orbital magnets and correlated states in magic-angle bilayer graphene, Nature (London) 574, 653 (2019). 
[43] A. W. W. Ludwig, M. P. A. Fisher, R. Shankar, and G. Grinstein, Integer quantum Hall transition: An alternative approach and exact results, Phys. Rev. B 50, 7526 (1994).

[44] G. J. Ferreira and D. Loss, Magnetically Defined Qubits on 3D Topological Insulators, Phys. Rev. Lett. 111, 106802 (2013).

[45] M. Z. Hasan and C. L. Kane, Colloquium: Topological insulators, Rev. Mod. Phys. 82, 3045 (2010).

[46] X.-L. Qi and S.-C. Zhang, Topological insulators and superconductors, Rev. Mod. Phys. 83, 1057 (2011).

[47] N. Read and D. Green, Paired states of fermions in two dimensions with breaking of parity and time-reversal symmetries and the fractional quantum Hall effect, Phys. Rev. B 61, 10267 (2000).

[48] I. Martin, Y. M. Blanter, and A. F. Morpurgo, Topological Confinement in Bilayer Graphene, Phys. Rev. Lett. 100, 036804 (2008).

[49] G. W. Semenoff, V. Semenoff, and F. Zhou, Domain Walls in Gapped Graphene, Phys. Rev. Lett. 101, 087204 (2008).

[50] C. Tauber, P. Delplace, and A. Venaille, Anomalous bulk-edge correspondence in continuous media, arXiv:1902.10050.

[51] B. Zhou, H.-Z. Lu, R.-L. Chu, S.-Q. Shen, and Q. Niu, Finite Size Effects on Helical Edge States in a Quantum Spin-Hall System, Phys. Rev. Lett. 101, 246807 (2008).

[52] K. Chang and W.-K. Lou, Helical Quantum States in HgTe Quantum Dots with Inverted Band Structures, Phys. Rev. Lett. 106, 206802 (2011).

[53] P. Potasz and J. Fernández-Rossier, Orbital magnetization of quantum spin Hall insulator nanoparticles, Nano Lett. 15, 5799 (2015).

[54] G. E. Volovik, An analog of the quantum Hall effect in a superfluid ${ }^{3}$ He film, J. Exp. Theor. Phys. 67, 1804 (1988).

[55] A. Souslov, K. Dasbiswas, M. Fruchart, S. Vaikuntanathan, and V. Vitelli, Topological Waves in Fluids with Odd Viscosity, Phys. Rev. Lett. 122, 128001 (2019).

[56] C. Tauber, P. Delplace, and A. Venaille, A bulk-interface correspondence for equatorial waves, J. Fluid Mech. 868, R2 (2019).

[57] W. Yao, S. A. Yang, and Q. Niu, Edge States in Graphene: From Gapped Flat-Band to Gapless Chiral Modes, Phys. Rev. Lett. 102, 096801 (2009).

[58] C. W. Groth, M. Wimmer, A. R. Akhmerov, and X. Waintal, Kwant: A software package for quantum transport, New J. Phys. 16, 063065 (2014).
[59] D. Jin, L. Lu, Z. Wang, C. Fang, J. D. Joannopoulos, M. Soljačić, L. Fu, and N. X. Fang, Topological magnetoplasmon, Nat. Commun. 7, 13486 (2016).

[60] S. Shen, Topological Insulators: Dirac Equation in Condensed Matters, Springer Series in Solid-State Sciences (Springer, Berlin, 2013).

[61] R. P. Feynman, Forces in molecules, Phys. Rev. 56, 340 (1939).

[62] Y. Zhao, J. Wyrick, F. D. Natterer, J. F. Rodriguez-Nieva, C. Lewandowski, K. Watanabe, T. Taniguchi, L. S. Levitov, N. B. Zhitenev, and J. A. Stroscio, Creating and probing electron whispering-gallery modes in graphene, Science 348, 672 (2015).

[63] J. F. Rodriguez-Nieva and L. S. Levitov, Berry phase jumps and giant nonreciprocity in Dirac quantum dots, Phys. Rev. B 94, 235406 (2016).

[64] M. Lee, J. R. Wallbank, P. Gallagher, K. Watanabe, T. Taniguchi, V. I. Fal'ko, and D. Goldhaber-Gordon, Ballistic miniband conduction in a graphene superlattice, Science $\mathbf{3 5 3}$, 1526 (2016).

[65] F. Ghahari, D. Walkup, C. Gutiérrez, J. F. Rodriguez-Nieva, Y. Zhao, J. Wyrick, F. D. Natterer, W. G. Cullen, K. Watanabe, T. Taniguchi, L. S. Levitov, N. B. Zhitenev, and J. A. Stroscio, An on/off Berry phase switch in circular graphene resonators, Science 356, 845 (2017).

[66] Y. Leviatan and A. Boag, Analysis of electromagnetic scattering from dielectric cylinders using a multifilament current model, IEEE Trans. Antennas Propag. 35, 1119 (1987).

[67] M. G. Imhof, Multiple multipole expansions for elastic scattering, J. Acoust. Soc. Am. 100, 2969 (1996).

[68] D. I. Kaklamani and H. T. Anastassiu, Aspects of the method of auxiliary sources (MAS) in computational electromagnetics, IEEE Antennas Propag. Mag. 44, 48 (2002).

[69] E. Moreno, D. Erni, C. Hafner, and R. Vahldieck, Multiple multipole method with automatic multipole setting applied to the simulation of surface plasmons in metallic nanostructures, J. Opt. Soc. Am. A 19, 101 (2002).

[70] G. Tayeb and S. Enoch, Combined fictitious-sourcesscattering-matrix method, J. Opt. Soc. Am. A 21, 1417 (2004).

[71] J. Gielis, A generic geometric transformation that unifies a wide range of natural and abstract shapes, Am. J. Bot. 90, 333 (2003). 